\title{
An Algebraic Representation of Graphs and Applications to Graph Enumeration
}

\author{
Ângela Mestre \\ Centro de Estruturas Lineares e Combinatórias, Universidade de Lisboa, Av. Prof. Gama Pinto 2, 1649-003 Lisboa, Portugal \\ Correspondence should be addressed to Ângela Mestre; mestre@cii.fc.ul.pt
}

Received 23 July 2012; Accepted 25 September 2012

Academic Editor: Xueliang Li

Copyright (c) 2013 Ângela Mestre. This is an open access article distributed under the Creative Commons Attribution License, which permits unrestricted use, distribution, and reproduction in any medium, provided the original work is properly cited.

We give a recursion formula to generate all the equivalence classes of connected graphs with coefficients given by the inverses of the orders of their groups of automorphisms. We use an algebraic graph representation to apply the result to the enumeration of connected graphs, all of whose biconnected components have the same number of vertices and edges. The proof uses Abel's binomial theorem and generalizes Dziobek's induction proof of Cayley's formula.

\section{Introduction}

As pointed out in [1], generating graphs may be useful for numerous reasons. These include giving more insight into enumerative problems or the study of some properties of graphs. Problems of graph generation may also suggest conjectures or point out counterexamples. The use of generating functions (or functionals) in the enumeration or generation of graphs is standard practice both in mathematics and physics [2-4]. However, this is by no means obligatory since any method of manipulating graphs may be used.

Furthermore, the problem of generating graphs taking into account their symmetries was considered as early as the 19th century [5] and more recently for instance, in [6]. In particular, in quantum field theory, generated graphs are weighted by scalars given by the inverses of the orders of their groups of automorphisms [4]. In [7, 8], this was handled for trees and connected multigraphs (with multiple edges and loops allowed), on the level of the symmetric algebra on the vector space of time-ordered field operators. The underlying structure is an algebraic graph representation subsequently developed in [9]. In this representation, graphs are associated with tensors whose indices correspond to the vertex numbers. In the former papers, this made it possible to derive recursion formulas to produce larger graphs from smaller ones by increasing by 1 the number of their vertices or the number of their edges. An interesting property of these formulas is that of satisfying alternative recurrences which relate either a tree or connected multigraph on $n$ vertices with all pairs of their connected subgraphs with total number of vertices equal to $n$. In the case of trees, the algorithmic description of the corresponding formula is about the same as that used by Dziobek in his induction proof of Cayley's formula $[10,11]$. Accordingly, the formula induces a recurrence for $n^{n-2} / n$ !, that is, the sum of the inverses of the orders of the groups of automorphisms of all the equivalence classes of trees on $n$ vertices [12, page 209].

For simplicity, here by graphs we mean simple graphs. However, our results generalize straightforwardly to graphs with multiple edges allowed. One instance of an algorithm for finding the biconnected components of a connected graph is given in [13]. Our goal here is rather to generate all the equivalence classes of connected graphs so that they are decomposed into their biconnected components and have the coefficients announced in the abstract. To this end, we give a suitable graph transformation to produce larger connected graphs from smaller ones by increasing the number of their biconnected components by one unit. This mapping is then used to extend the recurrence of [7] to connected graphs. This new recurrence decomposes the graphs into their biconnected components and, in addition, can be generalized to restricted classes of connected graphs with specified biconnected components. The proof proceeds as suggested in [7]. That is, given an arbitrary equivalence class whose 
representative is a graph on $m$ edges, say, $G$, we show that every one of the $m$ edges of the graph $G$ adds $1 /(m \cdot \mid$ aut $G \mid)$ to the coefficient of $G$. To this end, we use the fact that labeled vertices are held fixed under any automorphism.

Moreover, in the algebraic representation framework, the result yields a recurrence to generate linear combinations of tensors over the rational numbers. Each tensor represents a connected graph. As required, these linear combinations have the property that the sum of the coefficients of all the tensors representing isomorphic graphs is the inverse of the order of their group of automorphisms. In this context, tensors representing generated graphs are factorized into tensors representing their biconnected components. As in [7-9], a key feature of this result is its close relation to the algorithmic description of the computations involved. Indeed, it is easy to read off from this scheme not only algorithms to perform the computations, but even data structures relevant for an implementation.

Furthermore, we prove that when we only consider the restricted class of connected graphs whose biconnected components all have, say, $p$ vertices and $r$ edges, the corresponding recurrence has an alternative expression which relates connected graphs on $\mu$ biconnected components with all the $p$-tuples of their connected subgraphs with total number of biconnected components equal to $\mu-1$. This induces a recurrence for the sum of the inverses of the orders of the groups of automorphisms of all the equivalence classes of connected graphs on $\mu$ biconnected components with that property. The proof uses an identity related to Abel's binomial theorem $[14,15]$ and generalizes Dziobek's induction proof of Cayley's formula [10].

This paper is organized as follows. Section 2 reviews the basic concepts of graph theory underlying much of the paper. Section 3 contains the definitions of the elementary graph transformations to be used in the following. Section 4 gives a recursion formula for generating all the equivalence classes of connected graphs in terms of their biconnected components. Sections 5 and 6 review the algebraic representation and some of the linear mappings introduced in $[7,9]$. Section 7 derives an algebraic expression for the recurrence of Section 4 and for the particular case in which graphs are such that their biconnected components are all graphs on the same vertex and edge numbers. An alternative formulation for the latter is also given. Finally, Section 8 proves a Cayley-type formula for graphs of that kind.

\section{Basics}

We briefly review the basic concepts of graph theory that are relevant for the following sections. More details may be found in any standard textbook on graph theory such as [16].

Let $A$ and $B$ denote sets. By $[A]^{2}$ we denote the set of all the 2 -element subsets of $A$. Also, by $2^{A}$ we denote the power set of $A$, that is, the set of all the subsets of $A$. By card $A$ we denote the cardinality of the set $A$. Furthermore, we recall that the symmetric difference of the sets $A$ and $B$ is given by $A \Delta B:=(A \cup B) \backslash(A \cap B)$.

Here, a graph is a pair $G=(V, E)$, where $V \subset \mathbb{N}$ is a finite set and $E \subseteq[V]^{2}$. Thus, the elements of $E$ are 2-element subsets of $V$. The elements of $V$ and $E$ are called vertices and edges, respectively. In the following, the vertex set of a graph $G$ will often be referred to as $V(G)$, the edge set as $E(G)$. The cardinality of $V(G)$ is called the order of $G$, written as $|G|$. A vertex $v$ is said to be incident with an edge $e$ if $v \in e$. Then, $e$ is an edge at $v$. The two vertices incident with an edge are its endvertices. Moreover, the degree of a vertex $v$ is the number of edges at $v$. Two vertices $v$ and $u$ are said to be adjacent if $\{v, u\} \in E$. If all the vertices of $G$ are pairwise adjacent, then $G$ is said to be complete. A graph $G^{*}$ is called a subgraph of a graph $G$ if $V\left(G^{*}\right) \subseteq V(G)$ and $E\left(G^{*}\right) \subseteq E(G)$. A path is a graph $P$ on $n \geq 2$ vertices such that $E(P)=\left\{\left\{v_{1}, v_{2}\right\},\left\{v_{2}, v_{3}\right\}, \ldots,\left\{v_{n-1}, v_{n}\right\}\right\}, v_{j} \in V(P)$ for all $j=1, \ldots, n$. The vertices $v_{1}$ and $v_{n}$ have degree 1 , while the vertices $v_{2}, \ldots, v_{n-1}$ have degree 2 . In this context, the vertices $v_{1}$ and $v_{n}$ are linked by $P$ and called the endpoint vertices. The vertices $v_{2}, \ldots, v_{n-1}$ are called the inner vertices. A cycle is a graph $C$ on $n>2$ vertices such that $E(C)=$ $\left\{\left\{v_{1}, v_{2}\right\},\left\{v_{2}, v_{3}\right\}, \ldots,\left\{v_{n-1}, v_{n}\right\},\left\{v_{n}, v_{1}\right\}\right\}, v_{j} \in V(C)$ for all $j=1, \ldots, n$, every vertex having degree 2 . A graph is said to be connected if every pair of vertices is linked by a path. Otherwise, it is disconnected. Given a graph $G$, a maximal connected subgraph of $G$ is called a component of $G$. Furthermore, given a connected graph, a vertex whose removal (together with its incident edges) disconnects the graph is called a cutvertex. A graph that remains connected after erasing any vertex (together with incident edges) (resp. any edge) is said to be 2-connected (resp. 2-edge connected). A 2-connected graph (resp. 2-edge connected graph) is also called biconnected (resp. edge-biconnected). Given a connected graph $H$, a biconnected component of $H$ is a maximal subset of edges such that the induced subgraph is biconnected (see [17, Section 6.4] for instance). Here, we consider that an isolated vertex is, by convention, a biconnected graph with no biconnected components.

Moreover, given a graph $G$, the set $2^{E(G)}$ is a vector space over the field $\mathbb{Z}_{2}$ such that vector addition is given by the symmetric difference. The cycle space $\mathscr{C}(G)$ of the graph $G$ is defined as the subspace of $2^{E(G)}$ generated by all the cycles in $G$. The dimension of $\mathscr{C}(G)$ is called the cyclomatic number of the graph $G$. We recall that $\operatorname{dim} \mathscr{C}(G)=\operatorname{card} E(G)-|G|+c$, where $c$ denotes the number of connected components of the graph $G$ [18].

We now introduce a definition of labeled graph. Let $L$ be a finite set. Here, a labeling of a graph $G$ is a mapping $l: V(G) \rightarrow 2^{L}$ such that $\cup_{v \in V(G)} l(v)=L$ and $l(v) \cap l\left(v^{\prime}\right)=\emptyset$ for all $v, v^{\prime} \in V(G)$ with $v \neq v^{\prime}$. In this context, $L$ is called a label set, while the graph $G$ is said to be labeled with $L$ or simply a labeled graph. In the sequel, a labeling of a graph $G$ will be referred to as $l_{G}$. Moreover, an unlabeled graph is one labeled with the empty set.

Furthermore, an isomorphism between two graphs $G$ and $G^{*}$ is a bijection $\varphi: V(G) \rightarrow V\left(G^{*}\right)$ which satisfies the following conditions:

(i) $\left\{v, v^{\prime}\right\} \in E(G)$ if and only if $\left\{\varphi(v), \varphi\left(v^{\prime}\right)\right\} \in E\left(G^{*}\right)$,

(ii) $L \cap l_{G}(v)=L \cap l_{G^{*}}(\varphi(v))$. 
Clearly, an isomorphism defines an equivalence relation on graphs. In particular, an isomorphism of a graph $G$ onto itself is called an automorphism (or symmetry) of $G$.

\section{Elementary Graph Transformations}

We introduce the basic graph transformations to change the number of biconnected components of a connected graph by one unit.

Here, given an arbitrary set $X$, let $\mathbb{Q} X$ denote the free vector space on the set $X$ over $\mathbb{Q}$, the set of rational numbers. Also, for all integers $n \geq 1$ and $k \geq 0$ and label sets $L$, let

$$
\begin{gathered}
V^{n, k, L}=\{G:|G|=n, \operatorname{dim} \mathscr{C}(G)=k, G \text { is labeled } \\
\text { by } \left.l_{G}: V(G) \longrightarrow 2^{L}\right\} .
\end{gathered}
$$

Furthermore, let

(i) $V_{\text {conn }}^{n, k, L}=\left\{G \in V^{n, k, L}: G\right.$ is connected $\}$,

(ii) $V_{\text {biconn }}^{n, k, L}=\left\{G \in V^{n, k, L}: G\right.$ is biconnected $\}$.

In what follows, when $L=\emptyset$ we will omit $L$ from the upper indices in the previous definitions.

We proceed to the definition of the elementary linear mappings to be used in the following. Note that, for simplicity, our notation does not distinguish between two mappings defined both according to one of the following definitions, one on $V^{n, k, L}$ and the other on $V^{p, q, L^{\prime}}$ with $n \neq p$ or $k \neq q$ or $L \neq L^{\prime}$. This convention will often be used in the rest of the paper for all the mappings given in this section. Therefore, we will specify the domain of the mappings whenever confusion may arise.

(i) Adding a biconnected component to a connected graph: let $L$ be a label set. Let $G$ be a graph in $V_{\text {conn. }}^{n, k}$. Let $V(G)=\left\{v_{i}\right\}_{i=1, \ldots, n}$. For all $i=1, \ldots, n$, let $\mathscr{K}_{i}$ denote the set of biconnected components of $G$ such that $v_{i} \in V(H)$ for all $H \in \mathscr{K}_{i}$. Let $\mathscr{L}$ denote the set of all the ordered partitions of the set $\mathscr{K}_{i}$ into $p$ disjoint sets: $\mathscr{L}=\left\{z_{i}:=\left(\mathscr{K}_{i}^{(1)}, \ldots, \mathscr{K}_{i}^{(p)}\right): \cup_{l=1}^{p} \mathscr{K}_{i}^{(l)}\right.$ $=\mathscr{K}_{i}$ and $\mathscr{K}_{i}^{(l)} \cap \mathscr{K}_{i}^{\left(l^{\prime}\right)}=\emptyset \forall l, l^{\prime}=1, \ldots$, $p$ with $\left.l \neq l^{\prime}\right\}$. Furthermore, let $\mathscr{J}$ denote the set of all the ordered partitions of the set $l_{G}\left(v_{i}\right)$ into $p$ disjoint sets: $\mathscr{J}=\left\{w_{i}:=\left(l_{G}\left(v_{i}\right)^{(1)}, \ldots, l_{G}\left(v_{i}\right)^{(p)}\right)\right.$ : $\cup_{l=1}^{p} l_{G}\left(v_{i}\right)^{(l)}=l_{G}\left(v_{i}\right)$ and $l_{G}\left(v_{i}\right)^{(l)} \cap l_{G}\left(v_{i}\right)^{\left(l^{\prime}\right)}=\emptyset \forall l$, $l^{\prime}=1, \ldots, p$ with $\left.l \neq l^{\prime}\right\}$. Finally, let $\widehat{G}$ be a graph in $V_{\text {biconn }}^{p, q}$ such that $V(G) \cap V(\widehat{G})=\emptyset$. (In case the graph $\widehat{G}$ does not satisfy that property, we consider a graph $G^{\prime}$ instead such that $G^{\prime} \cong \widehat{G}$ and $V(G) \cap V\left(G^{\prime}\right)=\emptyset$. We will not point this out explicitly in the following.) Let $V(\widehat{G})=\left\{u_{l}\right\}_{l=1, \ldots, p}$. In this context, for all $i=1, \ldots, n$, define

$$
\begin{gathered}
r_{i}^{\widehat{G}}: \mathbb{Q} V_{\text {conn }}^{n, k, L} \longrightarrow \mathbb{Q} V_{\text {conn }}^{n+p-1, k+q, L} \\
G \longmapsto \sum_{z_{i} \in \mathscr{L} ; w_{i} \in \mathcal{J}} G_{w_{i}}^{z_{i}},
\end{gathered}
$$

where the graphs $G_{w_{i}}^{z_{i}}$ satisfy the following:

$$
\begin{aligned}
& \text { (a) } V\left(G_{w_{i}}^{z_{i}}\right)=V(G) \backslash\left\{v_{i}\right\} \cup V(\widehat{G}), \\
& \text { (b) } E\left(G_{w_{i}}^{z_{i}}\right)=E(\widehat{G}) \cup\left\{\{x, y\} \in E(G): v_{i} \notin\{x, y\}\right\}
\end{aligned}
$$

$$
\bigcup_{l=1}^{p}\left\{\left\{x, u_{l}\right\}:\left\{x, v_{i}\right\} \in E(G), x \in \cup_{H^{*} \in \mathscr{K}_{i}^{(l)}} V\left(H^{*}\right)\right\},
$$

$$
\begin{aligned}
& \text { (c) }\left.l_{G_{w_{i}}^{z_{i}}}\right|_{V(G) \backslash\left\{v_{i}\right\}}=\left.l_{G}\right|_{V(G) \backslash\left\{v_{i}\right\}} \text { and } l_{G_{w_{i}}^{z_{i}}}\left(u_{l}\right)=l_{G}\left(v_{i}\right)^{(l)} \\
& \text { for all } l=1, \ldots, p \text {. }
\end{aligned}
$$

The mappings $r_{i}^{\widehat{G}}$ are extended to all of $\mathbb{Q} V_{\text {conn }}^{n, k, L}$ by linearity. For instance, Figure 1 shows the result of applying the mapping $r_{i}^{C_{4}}$ to the cutvertex of a 2-edge connected graph with two biconnected components, where $C_{4}$ denotes a cycle on four vertices.

Furthermore, let $X \subseteq V_{\text {biconn. Given a linear }}^{p, q}$ combination of graphs $\vartheta=\sum_{G \in X} \alpha_{G} G$, where $\alpha_{G} \in \mathbb{Q}$, we define

$$
r_{i}^{\vartheta}:=\sum_{G \in X} \alpha_{G} r_{i}^{G}
$$

We proceed to generalize the edge contraction operation given in [16] to the operation of contracting a biconnected component of a connected graph.

(ii) Contracting a biconnected component of a connected graph: let $L$ be a label set. Let $G$ be a graph in $V_{\text {conn }}^{n, k, L}$. Let $\widehat{G} \in V_{\text {biconn }}^{p, q, L^{\prime}}$ be a biconnected component of $G$, where $L^{\prime}=\cup_{v \in V(\widehat{G})} l_{G}(v)$. Define

$$
c_{\widehat{G}}: V_{\text {conn }}^{n, k, L} \longrightarrow V_{\text {conn }}^{n-p+1, k-q, L} ; \quad G \longmapsto G^{*},
$$

where the graph $G^{*}$ satisfies the following:

(a) $V\left(G^{*}\right)=V(G) \backslash V(\widehat{G}) \cup\{v\}$, where $v:=\min \left\{v^{\prime} \epsilon\right.$ $\left.\mathbb{N}: v^{\prime} \notin V(G) \backslash V(\widehat{G})\right\}$

(b) $E\left(G^{*}\right)=\{\{x, y\} \in E(G):\{x, y\} \cap E(\widehat{G})=\emptyset\}$

$\cup\{\{x, v\}:\{x, y\} \in E(G) \backslash E(\widehat{G})$ and $y \in V(\widehat{G})\}$,

(c) $\left.l_{G^{*}}\right|_{V(G) \backslash V(\widehat{G})}=\left.l_{G}\right|_{V(G) \backslash V(\widehat{G})}$ and $l_{G^{*}}(v)=$
$\cup_{u \in V(\widehat{G})} l_{G}(u)$.

For instance, Figure 2 shows the result of applying the mapping $c_{C_{4}}$ to a 2-edge connected graph with three biconnected components.

We now introduce the following auxiliary mapping.

Let $L$ be a label set. Let $G$ be a graph in $V^{n, k, L}$. Let $V(G)=\left\{v_{i}\right\}_{i=1, \ldots, n}$. Let $L^{\prime}$ be a label set such that $L \cap L^{\prime}=\emptyset$. Also, let $\mathscr{I}$ denote the set of all the ordered partitions of the set $L^{\prime}$ into $n$ disjoint sets: $\mathscr{I}=\{y:=$ $\left(L^{\prime(1)}, \ldots, L^{\prime(n)}\right): \cup_{j=1}^{n} L^{\prime(j)}=L^{\prime}$ and $L^{\prime(i)} \cap L^{\prime(j)}=$ $\emptyset \forall i, j=1, \ldots, n$ with $i \neq j\}$. In this context, define

$$
\xi_{L^{\prime}}: \mathbb{Q} V^{n, k, L} \longrightarrow \mathbb{Q} V^{n, k, L \cup L^{\prime}} ; \quad G \longmapsto \sum_{y \in \mathscr{F}} G_{y},
$$



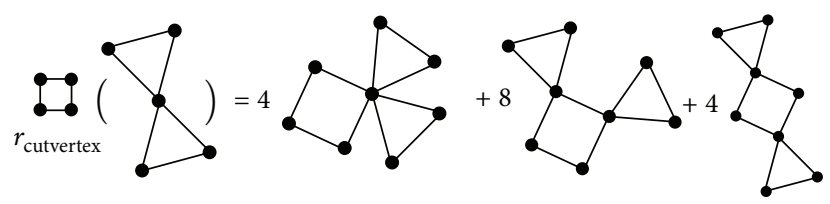

FIGURE 1: The linear combination of graphs obtained by applying the mapping $r_{i}^{C_{4}}$ to the cutvertex of the graph consisting of two triangles sharing one vertex.

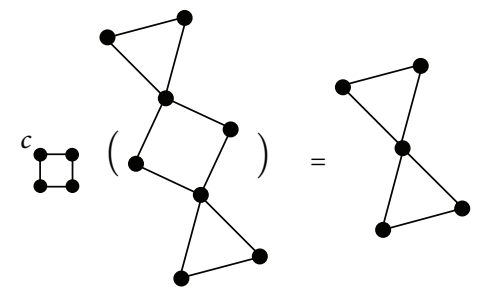

FIGURE 2: The graph obtained by applying the mapping $c_{C_{4}}$ to a graph with three biconnected components.

where the graphs $G_{y}$ satisfy the following:

(a) $V\left(G_{y}\right)=V(G)$,

(b) $E\left(G_{y}\right)=E(G)$,

(c) $l_{G_{y}}\left(v_{i}\right)=l_{G}\left(v_{i}\right) \cup L^{\prime(i)}$ for all $i=1, \ldots, n$ and $v_{i} \in V(G)$.

The mapping $\xi_{L^{\prime}}$ is extended to all of $\mathbb{Q} V^{n, k, L}$ by linearity.

\section{Generating Connected Graphs}

We give a recursion formula to generate all the equivalence classes of connected graphs. The formula depends on the vertex and cyclomatic numbers and produces larger graphs from smaller ones by increasing the number of their biconnected components by one unit. Here, graphs having the same parameters are algebraically represented by linear combinations over coefficients from the rational numbers. The key feature is that the sum of the coefficients of all the graphs in the same equivalence class is given by the inverse of the order of their group of automorphisms. Moreover, the generated graphs are automatically decomposed into their biconnected components.

In the rest of the paper, we often use the following notation: given a group $H$, by $|H|$ we denote the order of $H$. Given a graph $G$, by aut $G$ we denote the group of automorphisms of $G$. Accordingly, given an equivalence class $\mathscr{A}$, by aut $\mathscr{A}$ we denote the group of automorphisms of all the graphs in $\mathscr{A}$. Furthermore, given a set $W \subseteq V^{n, k, L}$, by $\mathscr{E}(W)$ we denote the set of equivalence classes of all the graphs in $W$.

We proceed to generalize the recursion formula for generating trees given in [7] to arbitrary connected graphs.

Theorem 1. For all $p>1$ and $q \geq 0$ suppose that $\beta_{\text {biconn }}^{p, q}:=$ $\sum_{G^{\prime} \in V_{\text {biconn }}^{p, q}} \sigma_{G^{\prime}} G^{\prime}$ with $\sigma_{G^{\prime}} \in \mathbb{Q}$, is such that for any equivalence class $\mathscr{A} \in \mathscr{E}\left(V_{\text {biconn }}^{p, q}\right)$ the following holds: (i) there exists $G^{\prime} \in \mathscr{A}$ such that $\sigma_{G^{\prime}}>0$, (ii) $\sum_{G^{\prime} \in \mathscr{A}} \sigma_{G^{\prime}}=1 / \mid$ aut $\mathscr{A} \mid$. In this context, given a label set $L$, for all $n \geq 1$ and $k \geq 0$, define $\beta_{\text {conn }}^{n, k, L} \in$ $\mathbb{Q} V_{\text {conn }}^{n, k, L}$ by the following recursion relation:

$$
\begin{gathered}
\beta_{\text {conn }}^{1,0, L}:=G, \quad \text { where } G=(\{1\}, \emptyset), l_{G}(1)=L, \\
\beta_{\text {conn }}^{1, k, L}:=0 \quad \text { if } k>0,
\end{gathered}
$$

$$
\begin{aligned}
\beta_{\text {conn }}^{n, k, L}:= & \frac{1}{k+n-1} \\
& \times \sum_{q=0}^{k} \sum_{p=2}^{n} \sum_{i=1}^{n-p+1}\left((q+p-1) r_{i}^{\beta_{\text {biconn }}^{p, q}}\left(\beta_{\text {conn }}^{n-p+1, k-q, L}\right)\right) .
\end{aligned}
$$

Then, $\beta_{\text {conn }}^{n, k, L}=\sum_{G \in V_{\text {conn }}^{n k, L}} \alpha_{G} G$ with $\alpha_{G} \in \mathbb{Q}$. Moreover, for any equivalence class $\mathscr{C} \in \mathscr{E}\left(V_{\text {conn }}^{n, k, L}\right)$, the following holds: (i) there exists $G \in \mathscr{C}$ such that $\alpha_{G}>0$, (ii) $\sum_{G \in \mathscr{C}} \alpha_{G}=1 / \mid$ aut $\mathscr{C} \mid$.

Proof. The proof is very analogous to the one given in [7] (see also $[8,19])$.

Lemma 2. Let $n \geq 1$ and $k \geq 0$ be fixed integers. Let $L$ be a label set. Let $\beta_{c o n n}^{n, k, L}=\sum_{G \in V_{c o n n}^{n, k, L}} \alpha_{G} G$ be defined by formula (8). Let $\mathscr{C} \in \mathscr{E}\left(V_{\text {conn }}^{n, k, L}\right)$ denote any equivalence class. Then, there exists $G \in \mathscr{C}$ such that $\alpha_{G}>0$.

Proof. The proof proceeds by induction on the number of biconnected components $\mu$. Clearly, the statement is true for $\mu=0$. We assume the statement to hold for all the equivalence classes in $\mathscr{E}\left(V_{\text {conn }}^{n-p+1, k-q, L}\right)$ with $p=2, \ldots, n-1$ and $q=$ $0, \ldots, k$, whose elements have $\mu-1$ biconnected components. Now, suppose that the elements of $\mathscr{C} \in \mathscr{E}\left(V_{\text {conn }}^{n, k, L}\right)$ have $\mu$ biconnected components. Let $\beta_{\text {biconn }}^{p, q q}=\sum_{G^{\prime} \in V_{\text {biconn }}^{p, q}} \sigma_{G^{\prime}} G^{\prime}$ with $\sigma_{G^{\prime}} \in \mathbb{Q}$. Recall that by (4) the mappings $r_{i}^{\beta_{\text {biconn }}^{\text {pq biconn }}}$ read as

$$
r_{i}^{\beta_{\text {biconn }}^{p, q}}:=\sum_{G^{\prime} \in V_{\text {biconn }}^{p, q}} \sigma_{G^{\prime}} r_{i}^{G^{\prime}} .
$$

Let $G$ denote any graph in $\mathscr{C}$. We proceed to show that a graph isomorphic to $G$ is generated by applying the mappings $r_{i}^{\beta_{\text {biconn }}^{p, q}}$ to a graph $G^{*} \in V_{\text {conn }}^{n-p+1, k-q, L}$ with $\mu-1$ biconnected components and such that $v_{G^{*}}>0$, where $v_{G^{*}}$ is the coefficient of $G^{*}$ in $\beta_{\text {conn }}^{n-p+1, k-q, L}$. Let $\widehat{G} \in V_{\text {biconn }}^{p, q, L^{\prime}}$ be any biconnected component of the graph $G$, where $L^{\prime}=\cup_{v \in V(\widehat{G})} l_{G}(v)$. 
Contracting the graph $\widehat{G}$ to the vertex $u:=\min \left\{u^{\prime} \in \mathbb{N}: u^{\prime} \notin\right.$ $V(G) \backslash V(\widehat{G})\}$ yields a graph $c_{\widehat{G}}(G) \in V_{\text {conn }}^{n-p+1, k-q, L}$ with $\mu-1$ biconnected components. Let $\mathscr{D} \in \mathscr{E}\left(V_{\text {conn }}^{n-p+1, k-q, L}\right)$ denote the equivalence class such that $c_{\widehat{G}}(G) \in \mathscr{D}$. By the inductive assumption, there exists a graph $H^{*} \in \mathscr{D}$ such that $H^{*} \cong$ $c_{\widehat{G}}(G)$ and $v_{H^{*}}>0$. Let $v_{j} \in V\left(H^{*}\right)$ be the vertex mapped to $u$ of $c_{\widehat{G}}(G)$ by an isomorphism. Relabeling the graph $\widehat{G}$ with the empty set yields a graph $G^{\prime} \in V_{\text {biconn }}^{p, q}$. Applying the mapping $r_{j}^{G^{\prime}}$ to the graph $H^{*}$ yields a linear combination of graphs, one of which is isomorphic to $G$. That is, there exists $H \cong G$ such that $\alpha_{H}>0$.

Lemma 3. Let $n \geq 1$ and $k \geq 0$ be fixed integers. Let $L$ be a label set such that cardL $\geq n$. Let $\beta_{\text {conn }}^{n, k, L}=\sum_{G \in V_{\text {conn }}^{n, L, L}} \alpha_{G} G$ be defined by formula (8). Let $\mathscr{C} \in \mathscr{E}\left(V_{\text {conn }}^{n, k, L}\right)$ be an equivalence class such that $l_{G}(v) \neq \emptyset$ for all $v \in V(G)$ and $G \in \mathscr{C}$. Then, $\sum_{G \in \mathscr{C}} \alpha_{G}=1$.

Proof. The proof proceeds by induction on the number of biconnected components $\mu$. Clearly, the statement is true for $\mu=0$. We assume the statement to hold for all the equivalence classes in $\mathscr{E}\left(V_{\text {conn }}^{n-p+1, k-q, L}\right)$ with $p=2, \ldots, n-1$ and $q=$ $0, \ldots, k$, whose elements have $\mu-1$ biconnected components and the property that no vertex is labeled with the empty set. Now, suppose that the elements of $\mathscr{C} \in \mathscr{E}\left(V_{\text {conn }}^{n, k, L}\right)$ have $\mu$ biconnected components. By Lemma 2, there exists a graph $G \in \mathscr{C}$ such that $\alpha_{G}>0$, where $\alpha_{G}$ is the coefficient of $G$ in $\beta_{\text {conn. }}^{n, k, L}$. Let $m:=\operatorname{card} E(G)$. Therefore, $m=k+n-1$. By assumption, $l_{G}(v) \neq \emptyset$ for all $v \in V(G)$ so that $\mid$ aut $\mathscr{C} \mid=1$. We proceed to show that $\sum_{G \in \mathscr{C}} \alpha_{G}=1$. To this end, we check from which graphs with $\mu-1$ biconnected components, the elements of $\mathscr{C}$ are generated by the recursion formula (8), and how many times they are generated.

Choose any one of the $\mu$ biconnected components of the graph $G \in \mathscr{C}$. Let this be the graph $\widehat{G} \in V_{\text {biconn }}^{p, q, L^{\prime}}$, where $L^{\prime}=\cup_{v \in V(\widehat{G})} l_{G}(v)$. Let $m^{\prime}:=\operatorname{card} E(\widehat{G})$ so that $m^{\prime}=$ $q+p-1$. Contracting the graph $\widehat{G}$ to the vertex $u$ with $u:=\min \left\{u^{\prime} \in \mathbb{N}: u^{\prime} \notin V(G) \backslash V(\widehat{G})\right\}$ yields a graph $c_{\widehat{G}}(G) \in V_{\text {conn }}^{n-p+1, k-q, L}$ with $\mu-1$ biconnected components. Let $\mathscr{D} \in \mathscr{E}\left(V_{\text {conn }}^{n-p+1, k-q, L}\right)$ denote the equivalence class containing $c_{\widehat{G}}(G)$. Since $l_{c_{\widehat{G}}(G)}(v) \neq \emptyset$ for all $v \in V\left(c_{\widehat{G}}(G)\right)$, we also have aut $\mathscr{D} \mid=1$. Let $\beta_{\text {conn }}^{n-p+1, k-q, L}=\sum_{G^{*} \in V_{\text {conn }}^{n-p+1, k q, L}} v_{G^{*}} G^{*}$ with $v_{G^{*}} \in \mathbb{Q}$. By Lemma 2, there exists a graph $H \in \mathscr{D}$ such that $H \cong c_{\widehat{G}}(G)$ and $v_{H}>0$. By the inductive assumption, $\sum_{G^{*} \in \mathscr{D}} v_{G^{*}}=1$. Now, let $v_{j} \in V(H)$ be the vertex which is mapped to $u$ of $c_{\widehat{G}}(G)$ by an isomorphism. Let $G^{\prime} \in V_{\text {biconn }}^{p, q}$ be the biconnected graph obtained by relabeling $\widehat{G}$ with the empty set. Also, let $\mathscr{A} \in \mathscr{E}\left(V_{\text {biconn }}^{p, q}\right)$ denote the equivalence class such that $G^{\prime} \in \mathscr{A}$. Apply the mapping $r_{j}^{G^{\prime}}$ to the graph $H$. Note that every one of the graphs in the linear combination $r_{j}^{G^{\prime}}(H)$ corresponds to a way of labeling the graph $G^{\prime}$ with $l_{c_{\bar{G}}(G)}(u)=L^{\prime}$. Therefore, there are |aut $\mathscr{A} \mid$ graphs in $r_{j}^{G^{\prime}}(H)$ which are isomorphic to the graph $G$. Since none of the vertices of the graph $H$ is labeled with the empty set, the mapping $r_{j}^{G^{\prime}}$ produces a graph isomorphic to $G$ from the graph $H$ with coefficient $\alpha_{G}^{*}=v_{H}>0$. Now, formula (8) prescribes to apply the mappings $r_{i}^{G^{\prime}}$ to the vertex which is mapped to $u$ by an isomorphism of every graph in the equivalence class $\mathscr{D}$ occurring in $\beta_{\text {conn }}^{n-p+1, k-q, L}$ (with non-zero coefficient). Therefore,

$$
\begin{aligned}
\sum_{G \in \mathscr{C}} \alpha_{G}^{*} & =\mid \text { aut } \mathscr{A} \mid \cdot \sum_{G^{*} \in \mathscr{D}} v_{G^{*}} \\
& =\mid \text { aut } \mathscr{A} \mid,
\end{aligned}
$$

where the factor $\mid$ aut $\mathscr{A} \mid$ on the right hand side of the first equality is due to the fact that every graph in the equivalence class $\mathscr{D}$ generates $\mid$ aut $\mathscr{A} \mid$ graphs in $\mathscr{C}$. Hence, according to formulas (8) and (4), the contribution to $\sum_{G \in \mathscr{C}} \alpha_{G}$ is $m^{\prime} / m$. Distributing this factor between the $m^{\prime}$ edges of the graph $\widehat{G}$ yields $1 / m$ for each edge. Repeating the same argument for every biconnected component of the graph $G$ proves that every one of the $m$ edges of the graph $G$ adds $1 / m$ to $\sum_{G \in \mathscr{C}} \alpha_{G}$. Hence, the overall contribution is exactly 1 . This completes the proof.

We now show that $\beta_{\text {conn }}^{n, k, L}$ satisfies the following property.

Lemma 4. Let $n \geq 1$ and $k \geq 0$ be fixed integers. Let $L$ and $L^{\prime}$ be label sets such that $L \cap L^{\prime}=\emptyset$. Then, $\beta_{\text {conn }}^{n, k, L \cup L^{\prime}}=\xi_{L^{\prime}}\left(\beta_{c o n n}^{n, k, L}\right)$.

Proof. Let $\xi_{L^{\prime}}: V^{n, k, L} \rightarrow V^{n, k, L \cup L^{\prime}}$ and $\tilde{\xi}_{L^{\prime}}: V^{n-p+1, k-q, L} \rightarrow$ $V^{n-p+1, k-q, L \cup L^{\prime}}$ be defined as in Section 3. The identity follows by noting that $\xi_{L^{\prime}} \circ r_{i}^{\beta_{\text {biconn }}^{p, q}}=r_{i}^{\beta_{\text {biconn }}^{p, q}} \circ \widetilde{\xi}_{L^{\prime}}$.

Lemma 5. Let $n \geq 1$ and $k \geq 0$ be fixed integers. Let $L$ be a label set. Let $\beta_{\text {conn }}^{n, k, L}=\sum_{G \in V_{\text {conn }}^{n, k}, \alpha_{G} G \text { be defined by formula }}$ (8). Let $\mathscr{C} \in \mathscr{E}\left(V_{\text {conn }}^{n, k, L}\right)$ denote any equivalence class. Then, $\sum_{G \in \mathscr{C}} \alpha_{G}=1 /|\operatorname{aut}(\mathscr{C})|$.

Proof. Let $G$ be a graph in $\mathscr{C}$. If $l_{G}(v) \neq \emptyset$ for all $v \in V(G)$, we simply recall Lemma 3 . Thus, we may assume that there exists a set $V^{\prime} \subseteq V(G)$ such that $l_{G}\left(V^{\prime}\right)=\{\emptyset\}$. Let $L^{\prime}$ be a label set such that $L \cap L^{\prime}=\emptyset$ and card $L^{\prime}=\operatorname{card} V^{\prime}$. Relabeling the graph $G$ with $L \cup L^{\prime}$ via the mapping $l: V(G) \rightarrow 2^{L \cup L^{\prime}}$ such that $l_{V(G) \backslash V^{\prime}}=\left.l_{G}\right|_{V(G) \backslash V^{\prime}}$ and $l(v) \neq \emptyset$ for all $v \in V^{\prime}$ yields a graph $G^{\prime} \in V_{\text {conn }}^{n, k, L \cup L^{\prime}}$ such that $\mid$ aut $G^{\prime} \mid=1$. Let $\mathscr{D} \in \mathscr{E}\left(V_{\text {conn }}^{n, k, L L^{\prime}}\right)$ be the equivalence class such that $G^{\prime} \in \mathscr{D}$. Let $\beta_{\text {conn }}^{n, k, L L^{\prime}}=\sum_{G^{\prime} \in V_{\text {conn }}^{n, k, L L^{\prime}}} v_{G^{\prime}} G^{\prime}$ with $v_{G^{\prime}} \in \mathbb{Q}$. By Lemma $3, \sum_{G^{\prime} \in \mathscr{D}} v_{G^{\prime}}=1$. Suppose now that there are exactly $T$ distinct labelings $l_{j}: V(G) \rightarrow 2^{L \cup L^{\prime}}, j=1, \ldots, T$ such that $\left.l_{j}\right|_{V(G) \backslash V^{\prime}}=\left.l_{G}\right|_{V(G) \mid V^{\prime}}, l_{j}(v) \neq \emptyset$ for all $v \in V^{\prime}$, and $G_{j} \in \mathscr{D}$, where $G_{j}$ is the graph obtained by relabeling $G$ with $l_{j}$. Clearly, $v_{G_{j}}=\alpha_{G}>0$, where $v_{G_{j}}$ is the coefficient of $G_{j}$ in $\beta_{\text {conn }}^{n, k, L L^{\prime}}$. Define $f_{j}: G \mapsto G_{j}$ for all $j=1, \ldots, T$. Now, repeating the 


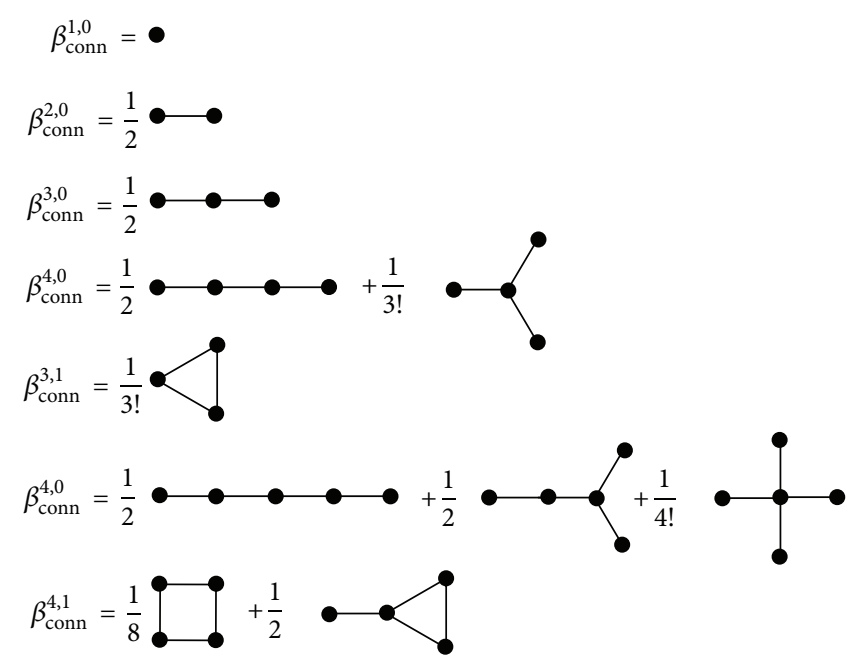

FIGURE 3: The result of computing all the pairwise non-isomorphic connected graphs as contributions to $\beta_{\text {conn }}^{n, k}$ via formula (8) up to order $n+k \leq 5$. The coefficients in front of graphs are the inverses of the orders of their groups of automorphisms.

same procedure for every graph in $\mathscr{C}$ and recalling Lemma 4 , we obtain

$$
\sum_{G^{\prime} \in \mathscr{D}} v_{G^{\prime}}=\sum_{j=1}^{T} \sum_{G \in \mathscr{C}} v_{f_{j}(G)}=T \sum_{G \in \mathscr{C}} \alpha_{G}=1 .
$$

That is, $\sum_{G \in \mathscr{C}} \alpha_{G}=1 / T$. Since $\mid$ aut $\mathscr{C} \mid=T$, we obtain $\sum_{G \in \mathscr{C}} \alpha_{G}=1 / \mid$ aut $(\mathscr{C}) \mid$.

This completes the proof of Theorem 1.

Figure 3 shows $\beta_{\text {conn }}^{n, k}$ for $1 \leq n+k \leq 5$. Now, given a connected graph $G$, let $\mathscr{V}_{G}$ denote the set of biconnected components of $G$. Given a set $X \subset \cup_{p=2}^{n} \cup_{q=0}^{k} V_{\text {biconn }}^{p, q}$, let $V_{X}^{n, k}:=$ $\left\{G \in V_{\text {conn }}^{n, k}: \mathscr{E}\left(\mathscr{V}_{G}\right) \subseteq \mathscr{E}(X)\right\}$ with the convention $V_{X}^{1,0}:=$ $\{(\{1\}, \emptyset)\}$. With this notation, Theorem 1 specializes straightforwardly to graphs with specified biconnected components.

Corollary 6. For all $p>1$ and $q \geq 0$ suppose that $\gamma_{\text {biconn }}^{p, q}:=$ $\sum_{G^{\prime} \in X^{p, q}} \sigma_{G^{\prime}} G^{\prime}$ with $\sigma_{G^{\prime}} \in \mathbb{Q}$ and $X^{p, q} \subseteq V_{b i c o n n}^{p, q}$, is such that for any equivalence class $\mathscr{A} \in \mathscr{E}\left(X^{p, q}\right)$, the following holds: (i) there exists $G^{\prime} \in \mathscr{A}$ such that $\sigma_{G^{\prime}}>0$, (ii) $\sum_{G^{\prime} \in \mathscr{A}} \sigma_{G^{\prime}}=$ $1 /$ aut $\mathscr{A} \mid$. In this context, for all $n \geq 1$ and $k \geq 0$, define $\gamma_{\text {conn }}^{n, k} \in$ $\mathbb{Q} V_{\text {conn }}^{n, k}$ by the following recursion relation:

$$
\begin{gathered}
\gamma_{\text {conn }}^{1,0}:=G, \quad \text { where } G=(\{1\}, \emptyset), \\
\gamma_{\text {conn }}^{1, k}:=0 \quad \text { if } k>0, \\
\gamma_{\text {conn }}^{n, k}:=\frac{1}{k+n-1} \\
\times \sum_{q=0}^{k} \sum_{p=2}^{n} \sum_{i=1}^{n-p+1}\left((q+p-1) r_{i}^{\gamma_{\text {biconn }}^{p, q}}\left(\gamma_{\text {conn }}^{n-p+1, k-q}\right)\right) .
\end{gathered}
$$

Then, $\gamma_{\text {conn }}^{n, k}=\sum_{G \in V_{X}^{n, k}} \alpha_{G} G$, where $\alpha_{G} \in \mathbb{Q}$ and $X:=$ $\cup_{p=2}^{n} \cup_{q=0}^{k} X^{p, q}$. Moreover, for any equivalence class $\mathscr{C} \in$ $\mathscr{E}\left(V_{X}^{n, k}\right)$ the following holds: (i) there exists $G \in \mathscr{C}$ such that $\alpha_{G}>0$ (ii) $\sum_{G \in \mathscr{C}} \alpha_{G}=1 / \mid$ aut $\mathscr{C} \mid$.

Proof. The result follows from the linearity of the mappings $r_{i}^{G}$ and the fact that larger graphs whose biconnected components are all in $X$ can only be produced from smaller ones with the same property.

\section{Algebraic Representation of Graphs}

We represent graphs by tensors whose indices correspond to the vertex numbers. Our description is essentially that of $[7,9]$. From the present section on, we will only consider unlabeled graphs.

Let $V$ be a vector space over $\mathbb{Q}$. Let $\mathrm{S}(V)$ denote the symmetric algebra on $V$. Then, $\mathrm{S}(V)=\bigoplus_{k=0}^{\infty} \mathrm{S}^{k}(V)$, where $\mathrm{S}^{0}(V):=\mathbb{Q} 1, \mathrm{~S}^{1}(V)=V$, and $\mathrm{S}^{k}(V)$ is generated by the free commutative product of $k$ elements of $V$. Also, let $\mathrm{S}(V)^{\otimes n}$ denote the $n$-fold tensor product of $\mathrm{S}(V)$ with itself. Recall that the multiplication in $\mathrm{S}(V)^{\otimes n}$ is given by the componentwise product:

$$
\begin{aligned}
& \cdot: \mathrm{S}(V)^{\otimes n} \times \mathrm{S}(V)^{\otimes n} \longrightarrow \mathrm{S}(V)^{\otimes n} ;\left(s_{1} \otimes \cdots \otimes s_{n}, s_{1}^{\prime} \otimes \cdots \otimes s_{n}^{\prime}\right) \\
& \quad \longmapsto s_{1} s_{1}^{\prime} \otimes \cdots \otimes s_{n} s_{n}^{\prime},
\end{aligned}
$$

where $s_{i}, s_{j}^{\prime}$ denote monomials on the elements of $V$ for all $i, j=1, \ldots, n$. We may now proceed to the correspondence between graphs on $\{1, \ldots, n\}$ and some elements of $\mathrm{S}(V)^{\otimes n}$. 

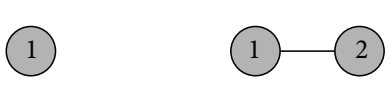

(a)

(b)

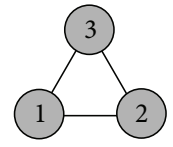

(c)
Figure 4: (a) An isolated vertex represented by $1 \in S(V)$. (b) A 2vertex tree represented by $R_{1,2} \in \mathrm{S}(V)^{\otimes 2}$. (c) A triangle represented by $R_{1,2} \cdot R_{1,3} \cdot R_{2,3} \in \mathrm{S}(V)^{\otimes 3}$.

First, for all $i, j=1, \ldots, n$ with $i \neq j$, we define the following tensors in $\mathrm{S}(V)^{\otimes n}$.

$$
R_{i, j}:=1^{\otimes i-1} \otimes v \otimes 1^{\otimes j-i-1} \otimes v \otimes 1^{\otimes n-j},
$$

where $v$ is any vector different from zero. (As in Section 3, for simplicity, our notation does not distinguish between elements, say, $R_{i, j} \in \mathrm{S}(V)^{\otimes n}$ and $R_{i, j} \in \mathrm{S}(V)^{\otimes n^{\prime}}$ with $n \neq n^{\prime}$. This convention will often be used in the rest of the paper for all the elements of the algebraic representation. Therefore, we will specify the set containing consider the given elements whenever necessary.) Now, for all $i, j=1, \ldots, n$ with $i \neq j$, let

(i) a tensor factor in the $i$ th position correspond to the vertex $i$ of a graph on $\{1, \ldots, n\}$,

(ii) a tensor $R_{i, j} \in \mathrm{S}(V)^{\otimes n}$ correspond to the edge $\{i, j\}$ of a graph on $\{1, \ldots, n\}$.

In this context, given a graph $G$ with $V(G)=\{1, \ldots, n\}$ and $E(G)=\left\{\left\{i_{k}, j_{k}\right\}\right\}_{k=1, \ldots, m}$, we define the following algebraic representation of graphs.

(a) If $m=0$, then $G$ is represented by the tensor $1 \otimes \cdots \otimes 1 \epsilon$ $\mathrm{S}(V)^{\otimes n}$.

(b) If $m>0$, then in $\mathrm{S}(V)^{\otimes n}$ the graph $G$ yields a monomial on the tensors which represent the edges of $G$. More precisely, since for all $1 \leq k \leq m$ each tensor $R_{i_{k}, j_{k}} \in \mathrm{S}(V)^{\otimes n}$ represents an edge of the graph $G$, this is uniquely represented by the following tensor $S_{1, \ldots, n}^{G} \in \mathrm{S}(V)^{\otimes n}$ given by the componentwise product of the tensors $R_{i_{k}, j_{k}}$ :

$$
S_{1, \ldots, n}^{G}:=\prod_{k=1}^{m} R_{i_{k}, j_{k}} .
$$

Figure 4 shows some examples of this correspondence. Furthermore, let $X \subseteq\left\{1, \ldots, n^{\prime}\right\}$ be a set of cardinality $n$ with $n^{\prime} \geq n$. Also, let $\sigma:\{1, \ldots, n\} \rightarrow X$ be a bijection. With this notation, define the following elements of $\mathrm{S}(V)^{\otimes n^{\prime}}$ :

$$
S_{\sigma(1), \ldots, \sigma(n)}^{G}:=\prod_{k=1}^{m} R_{\sigma\left(i_{k}\right), \sigma\left(j_{k}\right)} .
$$

In terms of graphs, the tensor $S_{\sigma(1), \ldots, \sigma(n)}^{G}$ represents a disconnected graph, say, $G^{\prime}$, on the set $\left\{1, \ldots, n^{\prime}\right\}$ consisting of a

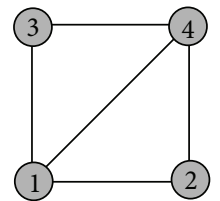

(a)

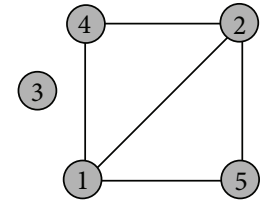

(b)
Figure 5: (a) The graph represented by the tensor $S_{1,2,3,4}^{G} \in \mathrm{S}(V)^{\otimes 4}$. (b) The graph represented by the tensor $S_{1,5,4,2}^{G} \in \mathrm{S}(V)^{\otimes 5}$ associated with $G$ and the bijection $\sigma:\{1,2,3,4\} \rightarrow\{1,2,4,5\} ; 1 \mapsto 1,2 \mapsto$ $5,3 \mapsto 4,4 \mapsto 2$.

graph isomorphic to $G$ whose vertex set is $X$ and $n^{\prime}-n$ isolated vertices in $\left\{1, \ldots, n^{\prime}\right\} \backslash X$. Figure 5 shows an example.

Furthermore, let $\mathrm{T}(\mathrm{S}(V))$ denote the tensor algebra on the graded vector space $\mathrm{S}(V): \mathrm{T}(\mathrm{S}(V)):=\bigoplus_{k=0}^{\infty} \mathrm{S}(V)^{\otimes k}$. In $\mathrm{T}(\mathrm{S}(V))$ the multiplication

$$
\text { - : } \mathrm{T}(\mathrm{S}(V)) \times \mathrm{T}(\mathrm{S}(V)) \longrightarrow \mathrm{T}(\mathrm{S}(V))
$$

is given by concatenation of tensors (e.g., see [20-22]):

$$
\begin{gathered}
\left(s_{1} \otimes \cdots \otimes s_{n}\right) \cdot\left(s_{1}^{\prime} \otimes \cdots \otimes s_{n^{\prime}}^{\prime}\right) \\
\quad:=s_{1} \otimes \cdots \otimes s_{n} \otimes s_{1}^{\prime} \otimes \cdots \otimes s_{n^{\prime}}^{\prime},
\end{gathered}
$$

where $s_{i}, s_{j}^{\prime}$ denote monomials on the elements of $V$ for all $i=1, \ldots, n$ and $j=1, \ldots, n^{\prime}$. We proceed to generalize the definition of the multiplication - to any two positions of the tensor factors. Let $\tau: \mathrm{S}(V)^{\otimes 2} \rightarrow \mathrm{S}(V)^{\otimes 2} ; s_{1} \otimes s_{2} \mapsto s_{2} \otimes s_{1}$. Moreover, define $\tau_{k}:=1^{\otimes k-1} \otimes \tau \otimes 1^{\otimes n-k-1}: \mathrm{S}(V)^{\otimes n} \rightarrow \mathrm{S}(V)^{\otimes n}$ for all $1 \leq k \leq n-1$. In this context, for all $1 \leq i \leq n$, $1 \leq j \leq n^{\prime}$, we define $\bullet_{i, j}: \mathrm{S}(V)^{\otimes n} \times \mathrm{S}(V)^{\otimes n^{\prime}} \rightarrow \mathrm{S}(V)^{\otimes n+n^{\prime}}$ by the following equation:

$$
\begin{aligned}
&\left(s_{1} \otimes \cdots \otimes s_{n}\right) \cdot{ }_{i, j}\left(s_{1}^{\prime} \otimes \cdots \otimes s_{n^{\prime}}^{\prime}\right) \\
&:=\left(\tau_{n-1} \circ \cdots \circ \tau_{i}\right)\left(s_{1} \otimes \cdots \otimes s_{n}\right) \\
& \otimes\left(\tau_{1} \circ \cdots \circ \tau_{j-1}\right)\left(s_{1}^{\prime} \otimes \cdots \otimes s_{n^{\prime}}^{\prime}\right) \\
&= s_{1} \otimes \cdots \otimes \widehat{s}_{i} \otimes \cdots \otimes s_{n} \otimes s_{i} \\
& \otimes s_{j}^{\prime} \otimes s_{1}^{\prime} \otimes \cdots \otimes \widehat{s_{j}^{\prime}} \otimes \cdots \otimes s_{n^{\prime}}^{\prime},
\end{aligned}
$$

where $\widehat{s}_{i}$ (resp. $\widehat{s_{j}^{\prime}}$ ) means that $s_{i}$ (resp. $s_{j}^{\prime}$ ) is excluded from the sequence. In terms of the tensors $S_{1, \ldots, n}^{G} \in \mathrm{S}(V)^{\otimes n}$ and $S_{1, \ldots, n^{\prime}}^{G^{\prime}} \in$ $\mathrm{S}(V)^{\otimes n^{\prime}}$ the equation previous yields

$$
S_{1, \ldots, n}^{G} \bullet i, j=S_{1, \ldots, n^{\prime}}^{G^{\prime}}:=S_{\sigma(1), \ldots, \sigma(n)}^{G} \cdot S_{\sigma^{\prime}(1), \ldots, \sigma^{\prime}\left(n^{\prime}\right)}^{G^{\prime}}
$$

where $S_{\sigma(1), \ldots, \sigma(n)}^{G}, S_{\sigma^{\prime}(1), \ldots, \sigma^{\prime}\left(n^{\prime}\right)}^{G^{\prime}} \in \mathrm{S}(V)^{\otimes n+n^{\prime}}, \sigma(k)=k$ if $1 \leq k<$ $i, \sigma(i)=n, \sigma(k)=k-1$ if $i<k \leq n$, and $\sigma^{\prime}(k)=k+n+1$ if $1 \leq k<j, \sigma^{\prime}(j)=n+1, \sigma^{\prime}(k)=k+n$ if $j<k \leq n^{\prime}$. 

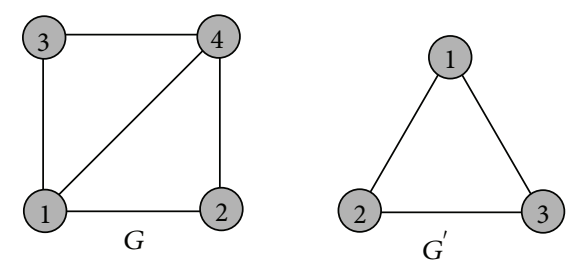

(a)

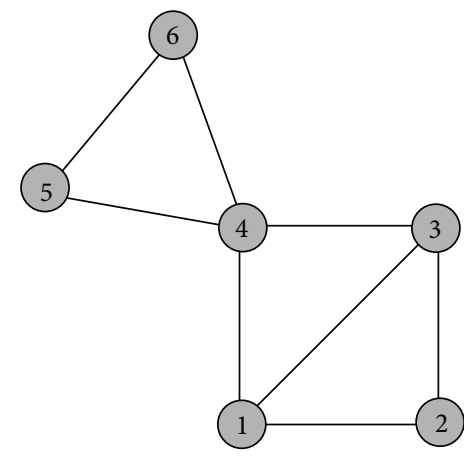

$H$

(b)

FIGURE 6: (a) The graphs represented by the tensors $S_{1,2,3,4}^{G} \in \mathrm{S}(V)^{\otimes 4}$ and $S_{1,2,3}^{G^{\prime}} \in \mathrm{S}(V)^{\otimes 3}$. (b) The graph represented by the tensor $S_{1, \ldots, 6}^{H}=$ $S_{1,2,3,4}^{G} \diamond_{3,2} S_{1,2,3}^{G^{\prime}} \in \mathrm{S}(V)^{\otimes 6}$.

Clearly, the tensor $S_{1, \ldots, n}^{G} \bullet_{i, j} S_{1, \ldots, n^{\prime}}^{G^{\prime}}$ represents a disconnected graph. Now, let $\cdot_{i}:=1^{\otimes i-1} \otimes \cdot \otimes 1^{\otimes n-i-1}: \mathrm{S}(V)^{\otimes n} \rightarrow \mathrm{S}(V)^{\otimes n-1}$. In $\mathrm{T}(\mathrm{S}(V))$, for all $1 \leq i \leq n, 1 \leq j \leq n^{\prime}$, define the following nonassociative and noncommutative multiplication:

$$
\diamond_{i, j}:={ }_{n} \circ \bullet_{i, j}: \mathrm{S}(V)^{\otimes n} \times \mathrm{S}(V)^{\otimes n^{\prime}} \longrightarrow \mathrm{S}(V)^{\otimes n+n^{\prime}-1}
$$

The tensor $S_{1, \ldots, n}^{G} \diamond_{i, j} S_{1, \ldots, n^{\prime}}^{G^{\prime}}$ represents the graph on $n+n^{\prime}-1$ vertices, say, $H$ obtained by gluing the vertex $i$ of the graph $G$ to the vertex $j$ of the graph $G^{\prime}$. If both $G$ and $G^{\prime}$ are connected, the vertex $n$ is clearly a cutvertex of the graph $H$. Figure 6 shows an example.

\section{Linear Mappings}

We recall some of the linear mappings given in [9].

Let $\mathscr{B}_{n, k} \subset \mathrm{S}(V)^{\otimes n}$ denote the vector space of all the tensors representing biconnected graphs in $V_{\text {biconn }}^{n, k}$. Let $\mathscr{B}:=$ $\mathscr{B}_{1,0} \bigoplus \mathscr{B}_{2,0} \bigoplus_{n=3}^{\infty} \bigoplus_{k=1}^{k_{\max }(n)} \mathscr{B}_{n, k} \subset \mathrm{T}(\mathrm{S}(V))$, where $k_{\text {max }}(n)=$ $(n(n-3) / 2)+1$ is, by Kirchhoff's lemma [18], the maximum cyclomatic number of a biconnected graph on $n$ vertices. Let the mapping $\Delta: \mathscr{B} \rightarrow \mathrm{T}(\mathrm{S}(V))$ be given by the following equations:

$$
\begin{gathered}
\Delta(1):=1 \otimes 1, \\
\Delta\left(B_{1, \ldots, n}^{G}\right):=\frac{1}{n} \sum_{i=1}^{n} \Delta_{i}\left(B_{1, \ldots, n}^{G}\right) \quad \text { if } n>1,
\end{gathered}
$$

where $G$ denotes a biconnected graph on $n$ vertices represented by $B_{1, \ldots, n}^{G} \in \mathrm{S}(V)^{\otimes n}$. To define the mappings $\Delta_{i}$, we introduce the following bijections:

$$
\begin{aligned}
& \text { (i) } \sigma_{i}: j \longmapsto \begin{cases}j & \text { if } 1 \leq j \leq i, \\
j+1 & \text { if } i+1 \leq j \leq n,\end{cases} \\
& \text { (ii) } v_{i}: j \longmapsto \begin{cases}j & \text { if } 1 \leq j \leq i-1, \\
j+1 & \text { if } i \leq j \leq n .\end{cases}
\end{aligned}
$$

In this context, for all $i=1, \ldots, n$ with $n>1$, the mappings $\Delta_{i}: \mathrm{S}(V)^{\otimes n} \rightarrow \mathrm{S}(V)^{\otimes n+1}$ are defined by the following equation:

$$
\begin{aligned}
\Delta_{i}\left(B_{1, \ldots, n}^{G}\right): & B_{\sigma_{i}(1), \ldots, \sigma_{i}(n)}^{G}+B_{v_{i}(1), \ldots, v_{i}(n)}^{G} \\
& =B_{1, \ldots, \widehat{i+1}, i+2, \ldots, n+1}^{G}+B_{1, \ldots, \hat{i}, i+1 \ldots, n+1}^{G}
\end{aligned}
$$

where $\hat{i}$ (resp., $\widehat{i+1}$ ) means that the index $i$ (resp., $i+1$ ) is excluded from the sequence. The tensor $B_{1, \ldots, \hat{i}, \ldots, n+1}^{G}$ (resp., $\left.B_{1, \ldots, \hat{i+1}, i+2, \ldots, n+1}^{G}\right)$ is constructed from $B_{1, \ldots, n}^{G}$ by transferring the monomial on the elements of $V$ which occupies the $k$ th tensor factor to the $(k+1)$ th position for all $i \leq k \leq n$ (resp., $i+1 \leq$ $k \leq n)$. Furthermore, suppose that $B_{\pi(1), \ldots, \pi(n)}^{G} \in \mathrm{S}(V)^{\otimes n^{\prime}}$ and that the bijection $\pi$ is such that $i \notin \pi(\{1, \ldots, n\}) \subset\left\{1, \ldots, n^{\prime}\right\}$. In this context, define

$$
\Delta_{i}\left(B_{\pi(1), \ldots, \pi(n)}^{G}\right):=B_{v_{i}(\pi(1)), \ldots, v_{i}(\pi(n))}^{G}
$$

in agreement with $\Delta(1):=1 \otimes 1$. It is straightforward to verify that the mappings $\Delta_{i}$ satisfy the following property:

$$
\Delta_{i} \circ \Delta_{i}=\Delta_{i+1} \circ \Delta_{i},
$$

where we used the same notation for $\Delta_{i}: \mathrm{S}(V)^{\otimes n} \rightarrow$ $\mathrm{S}(V)^{\otimes n+1}$ on the right of either side of the previous equation and $\Delta_{i}: \mathrm{S}(V)^{\otimes n+1} \rightarrow \mathrm{S}(V)^{\otimes n+2}$ as the leftmost operator on the left hand side of the equation. Accordingly, $\Delta_{i+1}$ : $\mathrm{S}(V)^{\otimes n+1} \rightarrow \mathrm{S}(V)^{\otimes n+2}$ as the leftmost operator on the right hand side of the equation.

Now, for all $m>0$, define the $m$ th iterate of $\Delta_{i}, \Delta_{i}^{m}$ : $\mathrm{S}(V)^{\otimes n} \rightarrow \mathrm{S}(V)^{\otimes n+m}$, recursively as follows:

$$
\begin{gathered}
\Delta_{i}^{1}:=\Delta_{i}, \\
\Delta_{i}^{m}:=\Delta_{i} \circ \Delta_{i}^{m-1},
\end{gathered}
$$

where $\Delta_{i}: \mathrm{S}(V)^{\otimes n+m-1} \rightarrow \mathrm{S}(V)^{\otimes n+m}$ in formula (28). This can be written in $m$ different ways corresponding to 
the composition of $\Delta_{i}^{m-1}$ with each of the mappings $\Delta_{j}$ : $\mathrm{S}(V)^{\otimes n+m-1} \rightarrow \mathrm{S}(V)^{\otimes n+m}$ with $i \leq j \leq i+m-1$. These are all equivalent by formula (26).

Extension to Connected Graphs. We now extend the mappings $\Delta_{i}$ to the vector space of all the tensors representing connected graphs $\mathscr{B}^{*}:=\bigoplus_{\mu=0}^{\infty} \mathscr{B}^{\diamond \mu}$, where $\mathscr{B}^{\diamond 0}=\mathbb{Q} 1$. We proceed to define $\mathscr{B}^{\diamond \mu}$ for $\mu>0$.

First, given two sets $A_{n} \subset \mathrm{S}(V)^{\otimes n}$ and $B_{p} \subset \mathrm{S}(V)^{\otimes p}$, by $A_{n} \diamond_{i, j} B_{p} \subset \mathrm{S}(V)^{\otimes n+p-1}$ with $i=1, \ldots, n, j=1, \ldots, p$ we denote the set of elements obtained by applying the mapping $\diamond_{i, j}$ to every ordered pair $\left(a \in A_{n}, b \in B_{p}\right)$. Also, let $\mathscr{B}_{2}:=$

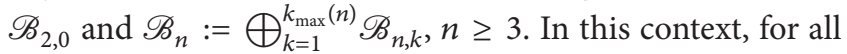
$\mu \geq 1$ and $n \geq \mu+1$, define $\mathscr{B}_{n}^{* \mu}$ as follows:

$$
\begin{gathered}
\mathscr{B}_{n}^{* \mu}=\bigcup_{n_{1}+\cdots+n_{\mu}=n+\mu-1} \bigcup_{i_{1}=1}^{n_{1}} \cdots \bigcup_{i_{\mu-1}=1}^{n_{\mu-1}} \bigcup_{j_{1}=1}^{n_{\mu}+\cdots+n_{2}-\mu+2} \cdots \bigcup_{j_{\mu-1}=1}^{n_{\mu}} \mathscr{B}_{n_{1}} \diamond_{i_{1}, j_{1}} \\
\left(\mathscr{B}_{n_{2}} \diamond_{i_{2}, j_{2}}\left(\cdots\left(\mathscr{B}_{n_{\mu-2}} \diamond_{i_{\mu-2}, j_{\mu-2}}\left(\mathscr{B}_{n_{\mu-1}} \diamond_{i_{\mu-1}, j_{\mu-1}} \mathscr{B}_{n_{\mu}}\right)\right) \cdots\right) .\right.
\end{gathered}
$$

Also, define

$$
\mathscr{B}_{n}^{\prime * \mu}=\bigcup_{\pi \in S_{n}}\left\{S_{\pi(1), \ldots, \pi(n)}^{G} \mid S_{1, \ldots, n}^{G} \in \mathscr{B}_{n}^{* \mu}\right\},
$$

where $S_{n}$ denotes the symmetric group on the set $\{1, \ldots, n\}$ and $S_{\pi(1), \ldots, \pi(n)}^{G}$ is given by formula (16). Finally, for all $\mu \geq 1$, define

$$
\mathscr{B}^{\diamond \mu}:=\bigoplus_{n=\mu+1}^{\infty} \mathscr{B}_{n}^{\prime * \mu}
$$

The elements of $\mathscr{B}^{\diamond \mu}$ are clearly tensors representing connected graphs on $\mu$ biconnected components. By (16) and (21), these may be seen as monomials on tensors representing biconnected graphs with the componentwise product $\cdot: \mathrm{S}(V)^{\otimes n} \times \mathrm{S}(V)^{\otimes n} \rightarrow \mathrm{S}(V)^{\otimes n}$ so that repeated indices correspond to cutvertices of the associated graphs. In this context, an arbitrary connected graph, say, $G$, on $n \geq 2$ vertices and $\mu \geq 1$ biconnected components yields

$$
\prod_{a=1}^{\mu} B_{\sigma_{a}(1), \ldots, \sigma_{a}\left(n_{a}\right)}^{G_{a}}
$$

Where, for all $1 \leq a \leq \mu, B_{\sigma_{a}(1), \ldots, \sigma_{a}\left(n_{a}\right)}^{G_{a}} \in \mathrm{S}(V)^{\otimes n}, G_{a}$ is a biconnected graph on $2 \leq n_{a} \leq n$ vertices represented by $B_{1, \ldots, n_{a}}^{G_{a}} \in \mathrm{S}(V)^{\otimes n_{a}}$, and $\sigma_{a}:\left\{1, \ldots, n_{a}\right\} \rightarrow X_{a} \subseteq\{1, \ldots, n\}$ is a bijection.

We now extend the mapping $\Delta:=(1 / n) \sum_{i=1}^{n} \Delta_{i}$ to $\mathscr{B}^{*}$ by requiring the mappings $\Delta_{i}$ to satisfy the following condition:

$$
\Delta_{i}\left(\prod_{a=1}^{\mu} B_{\sigma_{a}(1), \ldots, \sigma_{a}\left(n_{a}\right)}^{G_{a}}\right):=\prod_{a=1}^{\mu} \Delta_{i}\left(B_{\sigma_{a}(1), \ldots, \sigma_{a}\left(n_{a}\right)}^{G_{a}}\right) .
$$

Given a connected graph $G^{\prime}$, the mapping $\Delta_{i}$ may be thought of as a way of (a) splitting the vertex $i$ into two new vertices numbered $i$ and $i+1$ and (b) distributing the biconnected components sharing the vertex $i$ between the two new ones in all the possible ways. Analogously, the action of the mappings $\Delta_{i}^{m}$ consists of (a) splitting the vertex $i$ into $m+1$ new vertices numbered $i, i+1, \ldots, i+m$ and (b) distributing the biconnected components sharing the vertex $i$ between the $m+1$ new ones in all the possible ways.

We now combine the mappings $\Delta_{i}^{n-1}$ with tensors representing biconnected graphs. Let $n>1, p \geq 1$ and $1 \leq i \leq p$ be fixed integers. Let $\pi_{i}:\{1, \ldots, n\} \rightarrow\{i, i+1, \ldots, i+n-$ $1\} ; j \mapsto j+i-1$ be a bijection. Let $G$ be a biconnected graph on $n$ vertices represented by the tensor $B_{1, \ldots, n}^{G} \in$ $\mathrm{S}(V)^{\otimes n}$. The tensors $B_{i, i+1, \ldots, i+n-1}^{G}:=B_{\pi_{i}(1), \ldots, \pi_{i}(n)}^{G} \in \mathrm{S}(V)^{\otimes n+p-1}$ (see formula (16)) may be viewed as operators acting on $\mathrm{S}(V)^{\otimes n+p-1}$ by multiplication. In this context, consider the following mappings given by the composition of $B_{i, i+1, \ldots, i+n-1}^{G}$ with $\Delta_{i}^{n-1}$ :

$$
B_{i, i+1, \ldots, i+n-1}^{G} \circ \Delta_{i}^{n-1}: \mathrm{S}(V)^{\otimes p} \longrightarrow \mathrm{S}(V)^{\otimes n+p-1} .
$$

These are the analog of the mappings $r_{i}^{G}$ given in Section 3. In plain English, the mappings $B_{i, i+1, \ldots, i+n-1}^{G} \circ \Delta_{i}^{n-1}$ produce a connected graph with $n+p-1$ vertices from one with $p$ vertices in the following way:

(a) split the vertex $i$ into $n$ new vertices, namely, $i, i+1, \ldots$, $i+n-1$,

(b) distribute the biconnected components containing the split vertex between the $n$ new ones in all the possible ways,

(c) merge the $n$ new vertices into the graph $G$.

When the graph $G$ is a 2 -vertex tree, the mapping $R_{1,2} \circ \Delta$ coincides with the application $L=(\phi \otimes \phi) \Delta$ of [23] when $\phi$ acts on $\mathrm{S}(V)$ by multiplication with a vector.

To illustrate the action of the mappings $B_{\pi_{i}(1), \ldots, \pi_{i}(n)}^{G} \circ \Delta_{i}$, consider the graph $H$ consisting of two triangles sharing a vertex. Let this be represented by $B_{1,2,3}^{C_{3}} \cdot B_{3,4,5}^{C_{3}} \in \mathrm{S}(V)^{\otimes 5}$, where $C_{3}$ denotes a triangle represented by $B_{1,2,3}^{C_{3}}=R_{1,2} \cdot R_{2,3} \cdot R_{1,3} \epsilon$ $\mathrm{S}(V)^{\otimes 3}$. Let $T_{2}$ denote a 2-vertex tree represented by $B_{1,2}^{T_{2}}=$ $R_{1,2} \in \mathrm{S}(V)^{\otimes 2}$. Applying the mapping $R_{3,4} \circ \Delta_{3}$ to $H$ yields

$$
\begin{aligned}
R_{3,4} \circ \Delta_{3}\left(B_{1,2,3}^{C_{3}} \cdot B_{3,4,5}^{C_{3}}\right) \\
=R_{3,4} \cdot \Delta_{3}\left(B_{1,2,3}^{C_{3}}\right) \cdot \Delta_{3}\left(B_{3,4,5}^{C_{3}}\right) \\
=R_{3,4} \cdot\left(B_{1,2,3}^{C_{3}}+B_{1,2,4}^{C_{3}}\right) \cdot\left(B_{3,5,6}^{C_{3}}+B_{4,5,6}^{C_{3}}\right) \\
=R_{3,4} \cdot B_{1,2,3}^{C_{3}} \cdot B_{3,5,6}^{C_{3}}+R_{3,4} \cdot B_{1,2,3}^{C_{3}} \cdot B_{4,5,6}^{C_{3}} \\
\quad+R_{3,4} \cdot B_{1,2,4}^{C_{3}} \cdot B_{3,5,6}^{C_{3}}+R_{3,4} \cdot B_{1,2,4}^{C_{3}} \cdot B_{4,5,6}^{C_{3}} .
\end{aligned}
$$

Figure 7 shows the linear combination of graphs given by (35) after taking into account that the first and fourth terms as well as the second and third correspond to isomorphic 


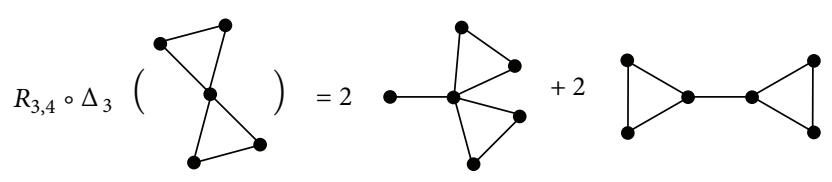

Figure 7: The linear combination of graphs obtained by applying the mapping $R_{3,4} \circ \Delta_{3}$ to the cutvertex of a graph consisting of two triangles sharing a vertex.

graphs. Note that 3 (resp. 4 ) is the only cutvertex of the graph represented by the first (resp. fourth) term, while 3 and 4 are both cutvertices of the graphs represented by the second or third terms.

\section{Further Recursion Relations}

Reference [7] gives two recursion formulas to generate all the equivalence classes of trees with coefficients given by the inverses of the orders of their groups of automorphisms. On the one hand, the main formula is such that larger trees are produced from smaller ones by increasing the number of their biconnected components by one unit. On the other hand, the alternative formula is such that for all $n \geq 2$, trees on $n$ vertices are produced by connecting a vertex of a tree on $i$ vertices to a vertex of a tree on $n-i$ vertices in all the possible ways. Theorem 1 generalizes the main formula to connected graphs. It is the aim of this section to derive an alternative formula for a simplified version of the latter.

Let $G$ denote a connected graph. Recall the notation introduced in Section $4 ; \mathscr{V}_{G}$ denotes the set of biconnected components of $G$ and $\mathscr{E}\left(\mathscr{V}_{G}\right)$ denotes the set of equivalence classes of the graphs in $\mathscr{V}_{G}$. Given a set $X \subset \cup_{p=2}^{n} \cup_{q=0}^{k} V_{\text {biconn }}^{p, q}$, let $V_{X}^{n, k}:=\left\{G \in V_{\text {conn }}^{n, k}: \mathscr{E}\left(\mathscr{V}_{G}\right) \subseteq \mathscr{E}(X)\right\}$ with the convention $V_{X}^{1,0}:=\{(\{1\}, \emptyset)\}$. With this notation, in the algebraic setting, Corollary 6 reads as follows.

Theorem 7. For all $p>1$ and $q \geq 0$, suppose that $\Phi_{1, \ldots, p}^{p, q}:=$ $\sum_{G^{\prime} \in X^{p, q}} \sigma_{G^{\prime}} B_{1, \ldots, p}^{G^{\prime}} \in \mathscr{B}_{p, q} \subset \mathrm{S}(V)^{\otimes p}$ with $\sigma_{G^{\prime}} \in \mathbb{Q}$ and $X^{p, q} \subseteq$ $V_{\text {biconn }}^{p, q}$, is such that for any equivalence class $\mathscr{A} \in \mathscr{E}\left(X^{p, q}\right)$ the following holds: (i) there exists $G^{\prime} \in \mathscr{A}$ such that $\sigma_{G^{\prime}}>0$, (ii) $\sum_{G^{\prime} \in \mathscr{A}} \sigma_{G^{\prime}}=1 /$ aut $\mathscr{A} \mid$. In this context, for all $n \geq 1$ and $k \geq 0$, define $\Psi^{n, k} \in \mathrm{S}(V)^{\otimes n}$ by the following recursion relation:

$$
\begin{aligned}
& \Psi^{1,0}:=1, \\
& \Psi^{1, k}:=0 \quad \text { if } k>0, \\
& \Psi^{n, k}:= \frac{1}{k+n-1} \\
& \times \sum_{q=0}^{k} \sum_{p=2}^{n} \sum_{i=1}^{n-p+1}\left((q+p-1) \Phi_{i, i+1, \ldots, i+p-1}^{p, q}\right. \\
&\left.\cdot \Delta_{i}^{p-1}\left(\Psi^{n-p+1, k-q}\right)\right) .
\end{aligned}
$$

Then, $\Psi^{n, k}=\sum_{G \in V_{X}^{n, k}} \alpha_{G} S_{1, \ldots, n}^{G}$, where $\alpha_{G} \in \mathbb{Q}$ and $X:=$ $\cup_{p=2}^{n} \cup_{q=0}^{k} X^{p, q}$. Moreover, for any equivalence class $\mathscr{C} \in$ $\mathscr{E}\left(V_{X}^{n, k}\right)$, the following holds: (i) there exists $G \in \mathscr{C}$ such that $\alpha_{G}>0$, (ii) $\sum_{G \in \mathscr{C}} \alpha_{G}=1 / \mid$ aut $\mathscr{C} \mid$.

Now, given a nonempty set $Y \subseteq V_{\text {biconn }}^{p, q}$, we use the following notation:

$V_{Y}^{\mu}:=\left\{G \in V_{\mathrm{conn}}^{(p-1) \mu+1, q \mu}: \mathscr{E}\left(\mathscr{V}_{G}\right) \subseteq \mathscr{E}(Y), \operatorname{card}\left(\mathscr{V}_{G}\right)=\mu\right\}$,

with the convention $V_{Y}^{0}:=\{(\{1\}, \emptyset)\}$. When we only consider graphs whose biconnected components are all in $Y$, the previous recurrence can be transformed into one on the number of biconnected components.

Theorem 8. Let $p>1$ and $q \geq 0$ be fixed integers. Let $Y \subseteq V_{b i c o n n}^{p, q}$ be a non-empty set. Suppose that $\phi_{1, \ldots, p}^{p, q}:=$ $\sum_{G^{\prime} \in Y} \sigma_{G^{\prime}} B_{1, \ldots, p}^{G^{\prime}} \in \mathscr{B}_{p, q} \subset \mathrm{S}(V)^{\otimes p}$ with $\sigma_{G^{\prime}} \in \mathbb{Q}$, is such that for any equivalence class $\mathscr{A} \in \mathscr{E}(Y)$ the following holds: (i) there exists $G^{\prime} \in \mathscr{A}$ such that $\sigma_{G^{\prime}}>0$, (ii) $\sum_{G^{\prime} \in \mathscr{A}} \sigma_{G^{\prime}}=$ $1 / \mid$ aut $(\mathscr{A}) \mid$. In this context, for all $\mu \geq 0$, define $\psi^{\mu} \in$ $\mathrm{S}(V)^{\otimes(p-1) \mu+1}$ by the following recursion relation:

$$
\begin{gathered}
\psi^{0}:=1 \\
\psi^{\mu}:=\frac{1}{\mu} \sum_{i=1}^{(p-1)(\mu-1)+1} \phi_{i, i+1, \ldots, i+p-1}^{p, q} \cdot \Delta_{i}^{p-1}\left(\psi^{\mu-1}\right) .
\end{gathered}
$$

Then, $\psi^{\mu}=\sum_{G \in V_{Y}^{\mu}} \alpha_{G} S_{1, \ldots,(p-1) \mu+1}^{G}$ with $\alpha_{G} \in \mathbb{Q}$. Moreover, for any equivalence class $\mathscr{C} \in \mathscr{E}\left(V_{Y}^{\mu}\right)$, the following holds: (i) there exists $G \in \mathscr{C}$ such that $\alpha_{G}>0$, (ii) $\sum_{G \in \mathscr{C}} \alpha_{G}=1 / \mid$ aut $(\mathscr{C}) \mid$.

Proof. In this case, $\Psi^{n, k}=0$ unless $n=(p-1) \mu+1$ and $k=q \mu$, where $\mu \geq 0$. Therefore, the recurrence of Theorem 7 can be easily converted into a recurrence on the number of biconnected components by setting $\psi^{\mu}:=\Psi^{(p-1) \mu+1, q \mu}$.

For $p=2$ and $q=0$, we recover the formula to generate trees of [7]. As in that paper and [8, 23], we may extend the result to obtain further interesting recursion relations. 
Proposition 9. For all $\mu>0$,

$$
\begin{gathered}
\psi^{\mu}=\frac{1}{\mu} \\
\times \sum_{i_{1}+\cdots+i_{p}=\mu-1}\left(\sum_{a_{1}=1}^{(p-1) i_{1}+1} \sum_{a_{2}=(p-1)\left(i_{1}+i_{2}\right)+2}\right. \\
\left.\ldots \sum_{a_{p}=(p-1)\left(i_{1}+\cdots+i_{p-1}\right)+p}^{(p-1)\left(i_{1}+\cdots+i_{p}\right)+p} \phi_{a_{1}, \ldots, a_{p}}^{p, q}\right) \\
\cdot\left(\psi^{i_{1}} \otimes \cdots \otimes \psi^{i_{p}}\right),
\end{gathered}
$$

where $i_{j}=0, \ldots, \mu-1$ for all $j=1, \ldots, p$.

Proof. Equation (40) is proved by induction on the number of biconnected components $\mu$. This is easily verified for $\mu=1$ :

$$
\psi^{1}=\phi_{1, \ldots p}^{p, q} \cdot(1 \otimes \cdots \otimes 1)=\phi_{1, \ldots p}^{p, q}
$$

We now assume the formula to hold for $\psi^{\mu-1}$. Then, formula (37) yields

$$
\begin{aligned}
\psi^{\mu}= & \frac{1}{\mu} \sum_{i=1}^{(p-1)(\mu-1)+1} \phi_{i, i+1, \ldots, i+p-1}^{p, q} \cdot \Delta_{i}^{p-1}\left(\psi^{\mu-1}\right) \\
= & \frac{1}{\mu(\mu-1)} \sum_{i=1}^{(p-1)(\mu-1)+1} \phi_{i, i+1, \ldots, i+p-1}^{p, q} \cdot \Delta_{i}^{p-1} \\
& \left(\sum_{i_{1}+\cdots+i_{p}=\mu-2}\left(\sum_{a_{1}=1}^{(p-1) i_{1}+1} \sum_{a_{2}=(p-1) i_{1}+2}^{(p-1)\left(i_{1}+i_{2}\right)+2} \sum_{(p-1)\left(i_{1}+\cdots+i_{p}\right)+p} \phi_{a_{1}, \ldots, a_{p}}^{p, q}\right)\right. \\
= & \frac{1}{\mu(\mu-1)} \sum^{a_{p}=(p-1)\left(i_{1}+\cdots+i_{p-1}\right)+p} \\
& \times \sum_{i_{1}+\cdots+i_{p}=\mu-2} \sum_{i=1}^{(p-1)(\mu-1)+1} \\
& \left(\Delta_{i}^{p-1}\left(\sum_{a_{1}=1}^{(p-1) i_{1}+1} \sum_{(p-1)\left(i_{1}+i_{2}\right)+2}^{i_{1}} \sum_{a_{2}=(p-1) i_{1}+2}^{i_{p}}\right)\right)
\end{aligned}
$$

$$
\begin{gathered}
\left.\ldots \sum_{a_{p}=(p-1)\left(i_{1}+\cdots+i_{p-1}\right)+p}^{(p-1)\left(i_{1}+\cdots+i_{p}\right)+p} \phi_{a_{1}, \ldots, a_{p}}^{p, q}\right) \\
=\frac{1}{\mu(\mu-1)} \\
\left.\times \phi_{i, i+1, \ldots, i+p-1}^{p, q} \cdot \Delta_{i}^{p-1}\left(\psi^{i_{1}} \otimes \cdots \otimes \psi^{i_{p}}\right)\right) \\
\times \sum_{i_{1}+\cdots+i_{p}=\mu-2}\left(\sum_{i=1}^{(p-1) i_{1}+1} \Delta_{i}^{p-1}\right.
\end{gathered}
$$$$
\left(\sum_{a_{1}=1}^{(p-1) i_{1}+1} \sum_{a_{2}=(p-1) i_{1}+2}^{(p-1)\left(i_{1}+i_{2}\right)+2}\right.
$$$$
\ldots \sum_{a_{p}=(p-1)\left(i_{1}+\cdots+i_{p-1}\right)+p}^{(p-1)\left(i_{1}+\cdots+i_{p}\right)+p}
$$$$
\left.\sum_{a_{p}=(p-1)\left(i_{1}+\cdots+i_{p-1}\right)+p}^{(p-1)\left(i_{1}+\cdots+i_{p}\right)+p} \phi_{a_{1}, \ldots, a_{p}}^{p, q}\right)
$$$$
\cdot \phi_{i, i+1, \ldots, i+p-1}^{p, q}
$$$$
\cdot\left(\Delta_{i}^{p-1}\left(\psi^{i_{1}}\right) \otimes \cdots \otimes \psi^{i_{p}}\right)
$$$$
+\sum_{i=(p-1) i_{1}+2}^{(p-1)\left(i_{1}+i_{2}\right)+2} \Delta_{i}^{p-1}
$$$$
\left(\sum_{a_{1}=1}^{(p-1) i_{1}+1} \sum_{a_{2}=(p-1) i_{1}+2}^{(p-1)\left(i_{1}+i_{2}\right)+2}\right.
$$$$
\left.\ldots \sum_{a_{p}=(p-1)\left(i_{1}+\cdots+i_{p-1}\right)+p}^{(p-1)\left(i_{1}+\cdots+i_{p}\right)+p}\right)
$$$$
\cdot \phi_{i, i+1, \ldots, i+p-1}^{p, q}
$$$$
\cdot\left(\psi^{i_{1}} \otimes \Delta_{i}^{p-1}\left(\psi^{i_{2}}\right) \otimes \cdots \otimes \psi^{i_{p}}\right)
$$$$
+\cdots+\sum_{i=(p-1)\left(i_{1}+i_{2}+\cdots+i_{p-1}\right)+p}^{(p-1)(i+1+\cdots+i p)+p} \Delta_{i}^{p-1}
$$$$
\sum_{a_{1}=1}^{(p-1) i_{1}+1} \sum_{a_{2}=(p-1) i_{1}+2}^{(p-1)\left(i_{1}+i_{2}\right)+2}
$$$$
\left.\ldots \sum_{a_{p}=(p-1)\left(i_{1}+\cdots+i_{p-1}\right)+p}^{(p-1)\left(i_{1}+\cdots+i_{p}\right)+p} \phi_{a_{1}, \ldots, a_{p}}^{p, q}\right)
$$$$
\cdot \phi_{i, i+1, \ldots, i+p-1}^{p, q}
$$ 


$$
\begin{aligned}
& \left.\left.\cdot\left(\psi^{i_{1}} \otimes \cdots \otimes \Delta_{i}^{p-1}\left(\psi^{i_{p}}\right)\right)\right)\right) \\
& =\frac{1}{\mu(\mu-1)} \\
& \times\left(\sum _ { i _ { 1 } + \cdots + i _ { p } = \mu - 2 } \left(\left(i_{1}+1\right)\right.\right. \\
& \times\left(\sum_{a_{1}=1}^{(p-1) i_{1}+p} \sum_{a_{2}=(p-1) i_{1}+p+1}^{(p-1)\left(i_{1}+i_{2}\right)+p+1}\right. \\
& \left.\ldots \sum_{a_{p}=(p-1)}^{(p-1)\left(i_{1}+\cdots+i_{p}\right)+2 p-1} \phi_{\left.i_{1}+\cdots+i_{p-1}\right)+2 p-1}^{p, q}{ }_{a_{1}, \ldots, a_{p}}\right) \\
& \cdot\left(\psi^{i_{1}+1} \otimes \cdots \otimes \psi^{i_{p}}\right)+\left(i_{2}+1\right) \\
& \times\left(\sum_{a_{1}=1}^{(p-1) i_{1}+1} \sum_{a_{2}=(p-1) i_{1}+2}^{(p-1)\left(i_{1}+i_{2}\right)+p+1}\right. \\
& \left.\cdots \sum_{a_{p}=(p-1)}^{(p-1)\left(i_{1}+\cdots+i_{p}\right)+2 p-1} \phi_{\left(i_{1}+\cdots+i_{p-1}\right)+2 p-1}^{p, q} \phi_{a_{1}, \ldots, a_{p}}\right) \\
& \cdot \phi_{i, i+1, \ldots, i+p-1}^{p, q} \cdot\left(\psi^{i_{1}} \otimes \psi^{i_{2}+1} \otimes \cdots \otimes \psi^{i_{p}}\right) \\
& +\cdots+\left(i_{p}+1\right) \\
& \times\left(\sum_{a_{1}=1}^{(p-1) i_{1}+1} \sum_{a_{2}=(p-1) i_{1}+2}^{(p-1)\left(i_{1}+i_{2}\right)+2}\right. \\
& \left.\ldots \sum_{a_{p}=(p-1)\left(i_{1}+\cdots+i_{p-1}\right)+2 p-1}^{(p-1)\left(i_{1}+\cdots+i_{p}\right)+p} \phi_{a_{1}, \ldots, a_{p}}^{p, q}\right) \\
& \left.\left.\cdot \phi_{i, i+1, \ldots, i+p-1}^{p, q} \cdot\left(\psi^{i_{1}} \otimes \cdots \otimes \psi^{i_{p}+1}\right)\right)\right) \\
& =\frac{1}{\mu(\mu-1)} \\
& \times\left(\sum_{i_{1}=0}^{\mu-1} \sum_{i_{2}+\cdots+i_{p}=\mu-1-i_{1}} i_{1}+\sum_{i_{2}=0}^{\mu-1} \sum_{i_{1}+i_{3}+\cdots+i_{p}=\mu-1-i_{2}} i_{2}\right. \\
& \left.+\cdots+\sum_{i_{p}=0}^{\mu-1} \sum_{i_{1}+\cdots+i_{p-1}=\mu-1-i_{p}} i_{p}\right) \\
& \times\left(\sum_{a_{1}=1}^{(p-1) i_{1}+1} \sum_{a_{2}=(p-1) i_{1}+2}^{(p-1)\left(i_{1}+i_{2}\right)+2}\right. \\
& \left.\cdots \sum_{a_{p}=(p-1)\left(i_{1}+\cdots+i_{p-1}\right)+p}^{(p-1)\left(i_{1}+\cdots+i_{p}\right)+p} \phi_{a_{1}, \ldots, a_{p}}^{p, q}\right)
\end{aligned}
$$

Note that formula (40) states that the linear combination of all the equivalence classes of connected graphs in $V_{Y}^{\mu}$ is given by summing over all the $p$-tuples of connected graphs with total number of biconnected components equal to $\mu-1$, gluing a vertex of each of them to distinct vertices of a graph in $\mathscr{E}(Y)$ in all the possible ways.

\section{Cayley-Type Formulas}

Let $Y \subseteq V_{\text {biconn }}^{p, q}$. Recall that $V_{Y}^{\mu}$ is the set of connected graphs on $\mu$ biconnected components, each of which has $p$ vertices and cyclomatic number $q$ and is isomorphic to a graph in $Y$. We proceed to use formula (40) to recover some enumerative results which are usually obtained via generating functions and the Lagrange inversion formula, see [3]. In particular, we extend to graphs in $V_{Y}^{\mu}$ the result that the sum of the inverses of the orders of the groups of automorphisms of all the pairwise nonisomorphic trees on $n$ vertices equals $n^{n-2} / n$ ! [12, page 209].

Proposition 10. Let $p>1$ and $q \geq 0$ be fixed integers. Let $Y \subseteq V_{\text {biconn }}^{p, q}$ be a non-empty set. For all $\mu \geq 0$,

$$
\sum_{\mathscr{C} \in \mathscr{E}\left(V_{Y}^{\mu}\right)} \frac{1}{|a u t \mathscr{C}|}=\frac{((p-1) \mu+1)^{\mu-2}}{\mu !}\left(p \sum_{\mathscr{A} \in \mathscr{E}(Y)} \frac{1}{|a u t \mathscr{A}|}\right)^{\mu} .
$$

Proof. We first recall the following well-known identities derived from Abel's binomial theorem [14]:

$$
\begin{gathered}
\sum_{i=1}^{n-1}\left(\begin{array}{c}
n \\
i
\end{array}\right) i^{i-1}(n-i)^{n-i-1}=2(n-1) n^{n-2}, \\
\sum_{i=0}^{n}\left(\begin{array}{c}
n \\
i
\end{array}\right)(x+i)^{i-1}(y+(n-i))^{n-i-1} \\
=\left(\frac{1}{x}+\frac{1}{y}\right)(x+y+n)^{n-1},
\end{gathered}
$$

where $x$ and $y$ are non-zero numbers. A proof may be found in [15] for instance. From (45) follows that for 
all $p>1$ and non-zero numbers $x_{1}, \ldots, x_{p}$, the identity

$$
\begin{gathered}
\sum_{i_{1}+\cdots+i_{p}=n}\left(\begin{array}{c}
n \\
i_{1}, \ldots, i_{p}
\end{array}\right)\left(x_{1}+i_{1}\right)^{i_{1}-1} \cdots\left(x_{p}+i_{p}\right)^{i_{p}-1} \\
=\frac{x_{1}+\cdots+x_{p}}{x_{1} \cdots x_{p}}\left(x_{1}+\cdots+x_{p}+n\right)^{n-1}
\end{gathered}
$$

holds. The proof proceeds by induction. For $p=2$ the identity specializes to (45). We assume the identity (46) to hold for $p-1$. Then,

$$
\begin{aligned}
\sum_{i_{1}+\cdots+i_{p}=n} & \left(\begin{array}{c}
n \\
i_{1}, \ldots, i_{p}
\end{array}\right)\left(x_{1}+i_{1}\right)^{i_{1}-1} \cdots\left(x_{p}+i_{p}\right)^{i_{p}-1} \\
= & \sum_{i_{1}=0}^{n} \sum_{i_{2}+\cdots+i_{p}=n-i_{1}}\left(\begin{array}{c}
n \\
i_{1}
\end{array}\right)\left(\begin{array}{c}
n-i_{1} \\
i_{2}, \ldots, i_{p}
\end{array}\right) \\
& \times\left(x_{1}+i_{1}\right)^{i_{1}-1} \cdots\left(x_{p}+i_{p}\right)^{i_{p}-1} \\
= & \frac{x_{2}+\cdots+x_{p}}{x_{2} \cdots x_{p}} \\
& \times \sum_{i_{1}=0}^{n}\left(\begin{array}{l}
n \\
i_{1}
\end{array}\right)\left(x_{1}+i_{1}\right)^{i_{1}-1}\left(x_{2}+\cdots+x_{p}+n-i_{1}\right)^{n-i_{1}-1} \\
= & \frac{x_{2}+\cdots+x_{p}}{x_{2} \cdots x_{p}} \\
& \times\left(\frac{1}{x_{1}}+\frac{1}{x_{2}+\cdots+x_{p}}\right)\left(x_{1}+\cdots+x_{p}+n\right)^{n-1} \\
= & \frac{x_{1}+\cdots+x_{p}}{x_{1} \cdots x_{p}}\left(x_{1}+\cdots+x_{p}+n\right)^{n-1} .
\end{aligned}
$$

We turn to the proof of formula (43). Let $I(\mu):=\sum_{\mathscr{C} \in \mathscr{C}\left(V_{Y}^{\mu}\right)}(1 /$ |aut $\mathscr{C} \mid$ ). Formula (40) induces the following recurrence for $I(\mu)$ :

$$
\begin{aligned}
I(0)= & 1, \\
I(\mu)= & \frac{1}{\mu} \sum_{\mathscr{A} \in \mathscr{E}(Y)} \frac{1}{\mid \text { aut } \mathscr{A} \mid} \\
& \times \sum_{i_{1}+\cdots+i_{p}=\mu-1}\left((p-1) i_{1}+1\right) \\
& \cdots\left((p-1) i_{p}+1\right) I\left(i_{1}\right) \cdots I\left(i_{p}\right) .
\end{aligned}
$$

We proceed to prove by induction that $I(\mu)=(((p-1) \mu+$ $\left.1)^{\mu-2} / \mu !\right)\left(p \sum_{\mathscr{A} \in \mathscr{C}(Y)}(1 / \mid \text { aut } \mathscr{A} \mid)\right)^{\mu}$. The result holds for $\mu=$ 0,1 :

$$
\begin{aligned}
& I(0)=1, \\
& I(1)=\sum_{\mathscr{A} \in \mathscr{E}(Y)} \frac{1}{\mid \text { aut } \mathscr{A} \mid} .
\end{aligned}
$$

We assume the result to hold for all $0 \leq i \leq \mu-1$. Then,

$$
\begin{aligned}
I(\mu)= & \frac{1}{\mu_{i_{1}+\cdots+i_{p}=\mu-1}}\left((p-1) i_{1}+1\right) \\
& \cdots\left((p-1) i_{p}+1\right) I\left(i_{1}\right) \cdots I\left(i_{p}\right) \sum_{\mathscr{A} \in \mathscr{E}(Y)} \frac{1}{\mid \text { aut } \mathscr{A} \mid}
\end{aligned}
$$

$$
\begin{aligned}
= & \frac{p^{\mu-1}}{\mu} \sum_{i_{1}+\cdots+i_{p}=\mu-1} \frac{1}{i_{1} ! \cdots i_{p} !}\left((p-1) i_{1}+1\right)^{i_{1}-1} \\
& \cdots\left((p-1) i_{p}+1\right)^{i_{p}-1}\left(\sum_{\mathscr{A} \in \mathscr{E}(Y)} \frac{1}{\mid \text { aut } \mathscr{A} \mid}\right)^{\mu} \\
= & \frac{p^{\mu-1}}{\mu !}(p-1)^{\mu-1-p}
\end{aligned}
$$$$
\times \sum_{i_{1}+\cdots+i_{p}=\mu-1}\left(\begin{array}{c}
\mu-1 \\
i_{1}, \ldots, i_{p}
\end{array}\right)\left(i_{1}+\frac{1}{p-1}\right)^{i_{1}-1}
$$

$$
\cdots\left(i_{p}+\frac{1}{p-1}\right)^{i_{p}-1}\left(\sum_{\mathscr{A} \in \mathscr{E}(Y)} \frac{1}{\mid \text { aut } \mathscr{A} \mid}\right)^{\mu}
$$$$
=\frac{1}{\mu !}(p-1)^{\mu-1-p}(p-1)^{p-1}
$$$$
\times\left(\mu-1+\frac{p}{p-1}\right)^{\mu-2}\left(p \sum_{\mathscr{A} \in \mathscr{E}(Y)} \frac{1}{\mid \text { aut } \mathscr{A} \mid}\right)^{\mu}
$$

$$
=\frac{1}{\mu !}((p-1) \mu+1)^{\mu-2}\left(p \sum_{\mathscr{A} \in \mathscr{E}(Y)} \frac{1}{\mid \text { aut } \mathscr{A} \mid}\right)^{\mu},
$$

where we used formula (46) in going from (52) to (53). This completes the proof of Proposition 10.

The corollary is now established.

Corollary 11. Let $p>1$ and $q \geq 0$ be fixed integers. Let $Y \subseteq$ $V_{\text {biconn }}^{p, q}$ be a non-empty set. For all $\mu \geq 0,\left\{G \in V_{Y}^{\mu} \mid V(G)=\right.$ $\{1, \ldots,(p-1) \mu+1\}$ is a set of cardinality

$$
\frac{((p-1) \mu+1) !((p-1) \mu+1)^{\mu-2}}{\mu !}\left(p \sum_{\mathscr{A} \in \mathscr{E}(Y)} \frac{1}{|a u t \mathscr{A}|}\right)^{\mu} .
$$

Proof. The result is a straightforward application of Lagrange's theorem to the symmetric group on the set $\{1, \ldots,(p-1) \mu+1\}$ and each of its subgroups aut $\mathscr{C}$, for all $\mathscr{C} \in \mathscr{E}\left(V_{Y}^{\mu}\right)$.

For $p=2$ and $q=0$, formula (55) specializes to Cayley's formula [11]:

$$
(\mu+1)^{\mu-1}=n^{n-2}
$$


where $n=\mu+1$ is the number of vertices of all the trees with $\mu$ biconnected components. In this particular case, the recurrence given by formula (48) can be easily transformed into a recurrence on the number of vertices $n$ :

$$
\begin{aligned}
& J(1)=1, \\
& J(n)=\frac{1}{2(n-1)} \sum_{i=1}^{n-1} i(n-i) J(i) J(n-i),
\end{aligned}
$$

which by (44) yields $J(n)=n^{n-2} / n$ !. Now, for $T(n)=n ! J(n)$, we obtain Dziobek's recurrence for Cayley's formula [10, 24]:

$$
\begin{aligned}
& T(1)=1 \\
& T(n)=\frac{1}{2(n-1)} \sum_{i=1}^{n-1}\left(\begin{array}{c}
n \\
i
\end{array}\right) i(n-i) T(i) T(n-i) .
\end{aligned}
$$

Furthermore, for graphs whose biconnected components are all complete graphs on $p$ vertices, formula (55) yields

$$
\frac{((p-1) \mu+1) !((p-1) \mu+1)^{\mu-2}}{(p-1) !^{\mu} \mu !}
$$

in agreement with Husimi's result for this particular case [25] (see also [26]). Also, when the biconnected components are all cycles of length $p$, we recover a particular case of Leroux's result [27]:

$$
\frac{((p-1) \mu+1) !((p-1) \mu+1)^{\mu-2}}{2^{\mu} \mu !}
$$

\section{Acknowledgment}

The research was supported through the fellowship SFRH/ BPD/48223/2008 provided by the Portuguese Foundation for Science and Technology (FCT).

\section{References}

[1] R. C. Read, "A survey of graph generation techniques," in Combinatorial Mathematics 8, vol. 884 of Lecture Notes in Math., pp. 77-89, Springer, Berlin, Germany, 1981.

[2] F. Harary and E. M. Palmer, Graphical Enumeration, Academic Press, New York, NY, USA, 1973.

[3] F. Bergeron, G. Labelle, and P. Leroux, Combinatorial Species and Tree-Like Structures, vol. 67, Cambridge University Press, Cambridge, UK, 1998.

[4] C. Itzykson and J. B. Zuber, Quantum Field Theory, McGrawHill, New York, NY, USA, 1980.

[5] C. Jordan, "Sur les assemblages de lignes," Journal für die Reine und Angewandte Mathematik, vol. 70, pp. 185-190, 1869.

[6] R. C. Read, "Every one a winner or how to avoid isomorphism search when cataloguing combinatorial configurations. Algorithmic aspects of combinatorics (Conference in Vancouver Island, BC, Canada, 1976)," Annals of Discrete Mathematics, vol. 2, pp. 107-120, 1978.

[7] Â. Mestre and R. Oeckl, "Combinatorics of $n$-point functions via Hopf algebra in quantum field theory," Journal of Mathematical Physics, vol. 47, no. 5, p. 052301, 16, 2006.
[8] Â. Mestre and R. Oeckl, "Generating loop graphs via Hopf algebra in quantum field theory," Journal of Mathematical Physics, vol. 47, no. 12, Article ID 122302, p. 14, 2006.

[9] Â. Mestre, "Combinatorics of 1-particle irreducible $n$-point functions via coalgebra in quantum field theory," Journal of Mathematical Physics, vol. 51, no. 8, Article ID 082302, 2010.

[10] O. Dziobek, "Eine formel der substitutionstheorie," Sitzungsberichte der Berliner Mathematischen Gesellschaft, vol. 17, pp. 64-67, 1947.

[11] A. Cayley, "A theorem on trees," Quarterly Journal of Pure and Applied Mathematics, vol. 23, pp. 376-378, 1889.

[12] G. Pólya, "Kombinatorische Anzahlbestimmungen für Gruppen, Graphen und chemische Verbindungen," Acta Mathematica, vol. 68, pp. 145-254, 1937.

[13] R. Tarjan, "Depth-first search and linear graph algorithms," SIAM Journal on Computing, vol. 1, no. 2, pp. 146-160, 1972.

[14] N. Abel, "Beweis eines Ausdruckes, von welchem die BinomialFormel ein einzelner Fall ist," Journal für die Reine und Angewandte Mathematik, vol. 1, pp. 159-160, 1826.

[15] L. Székely, "Abel's binomial theorem," http://www.math.sc .edu/ szekely/abel.pdf.

[16] R. Diestel, Graph Theory, vol. 173, Springer, Berlin, Germany, 5rd edition, 2005.

[17] M. J. Atallah and S. Fox, Algorithms and Theory of Computation Handbook, CRC Press, Boca Raton, Fla, USA, 1998.

[18] G. Kirchhoff, "Über die Auflösung der Gleichungen, auf welche man bei der Untersuchung der linearen Vertheilung galvanischer Ströme geführt wird," Annual Review of Physical Chemistry, vol. 72, pp. 497-508, 1847.

[19] Â. Mestre, "Generating connected and 2-edge connected graphs," Journal of Graph Algorithms and Applications, vol. 13, no. 2, pp. 251-281, 2009.

[20] J. Cuntz, R. Meyer, and J. M. Rosenberg, Topological and Bivariant K-Theory, vol. 36, Birkhäuser, Basel, Switzerland, 2007.

[21] C. Kassel, Quantum Groups, vol. 155, Springer, New York, NY, USA, 1995.

[22] J.-L. Loday, Cyclic Homology, vol. 301, Springer, Berlin, Germany, 1992.

[23] M. Livernet, "A rigidity theorem for pre-Lie algebras," Journal of Pure and Applied Algebra, vol. 207, no. 1, pp. 1-18, 2006.

[24] J. W. Moon, "Various proofs of Cayley's formula for counting trees," in A Seminar on Graph Theory, pp. 70-78, Holt, Rinehart \& Winston, New York, NY, USA, 1967.

[25] K. Husimi, "Note on Mayers' theory of cluster integrals," The Journal of Chemical Physics, vol. 18, pp. 682-684, 1950.

[26] J. Mayer, "Equilibrium statistical mechanics," in The International Encyclopedia of Physical Chemistry and Chemical Physics, Pergamon Press, Oxford, UK, 1968.

[27] P. Leroux, “Enumerative problems inspired by Mayer's theory of cluster integrals," Electronic Journal of Combinatorics, vol. 11, no. 1, research paper 32, p. 28, 2004. 


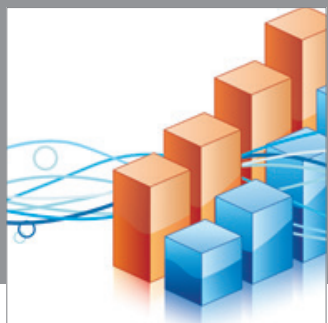

Advances in

Operations Research

mansans

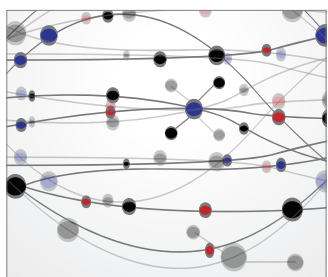

The Scientific World Journal
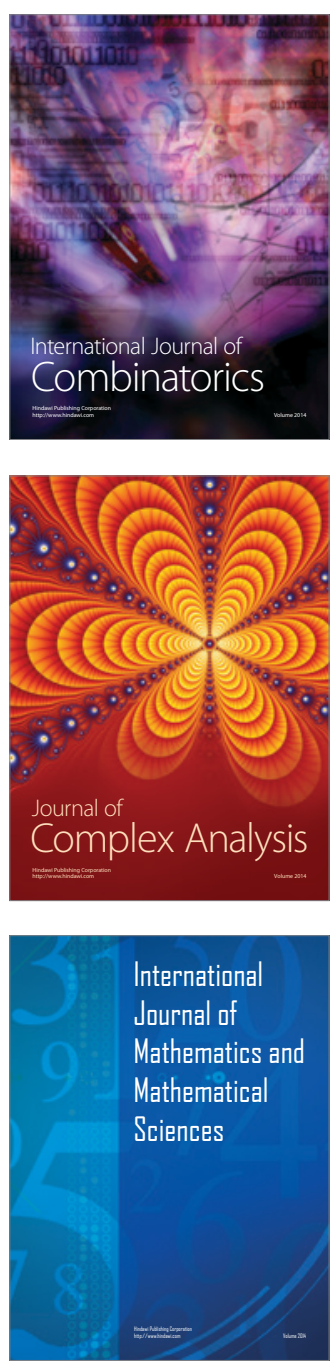
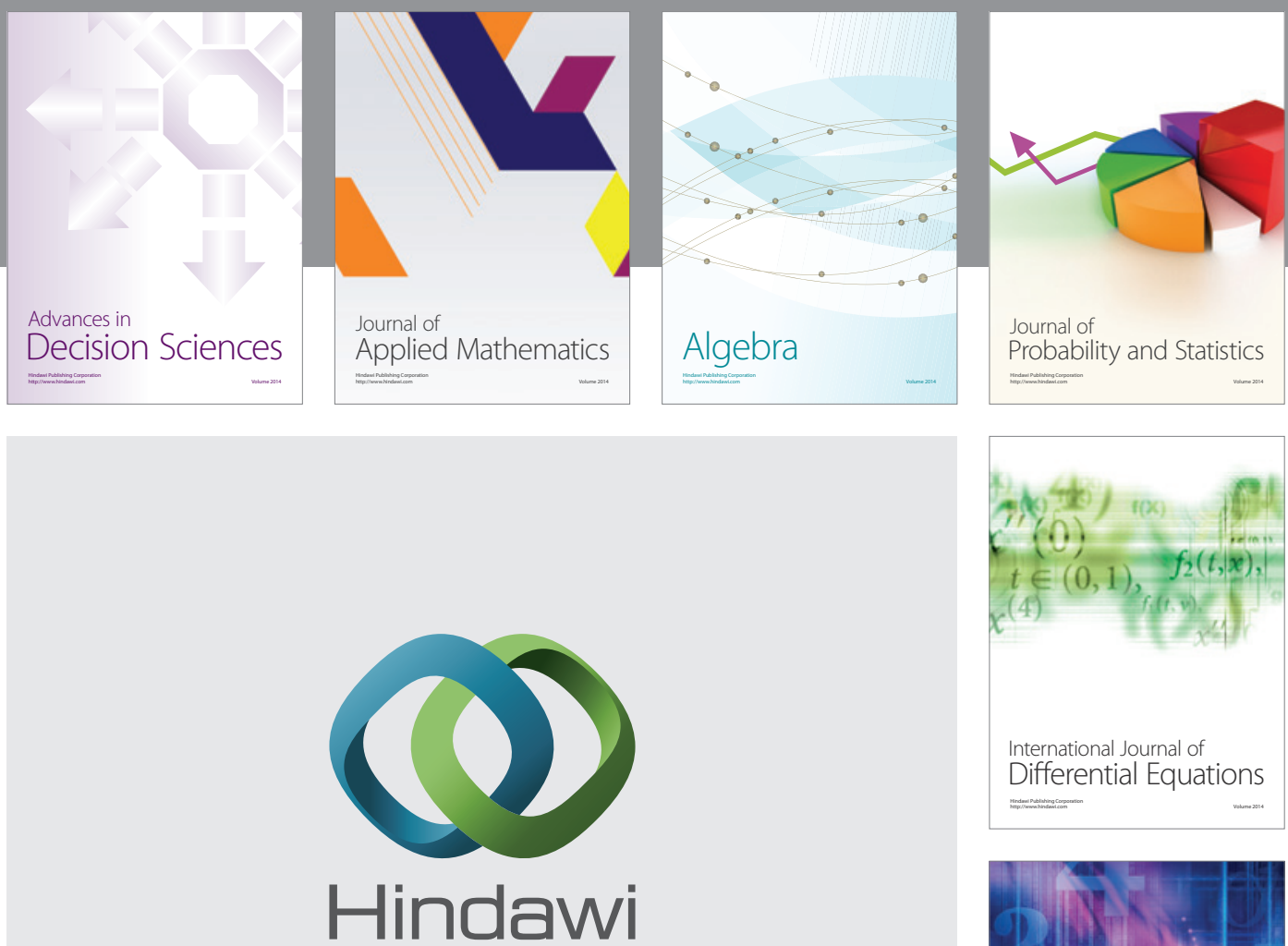

Submit your manuscripts at http://www.hindawi.com
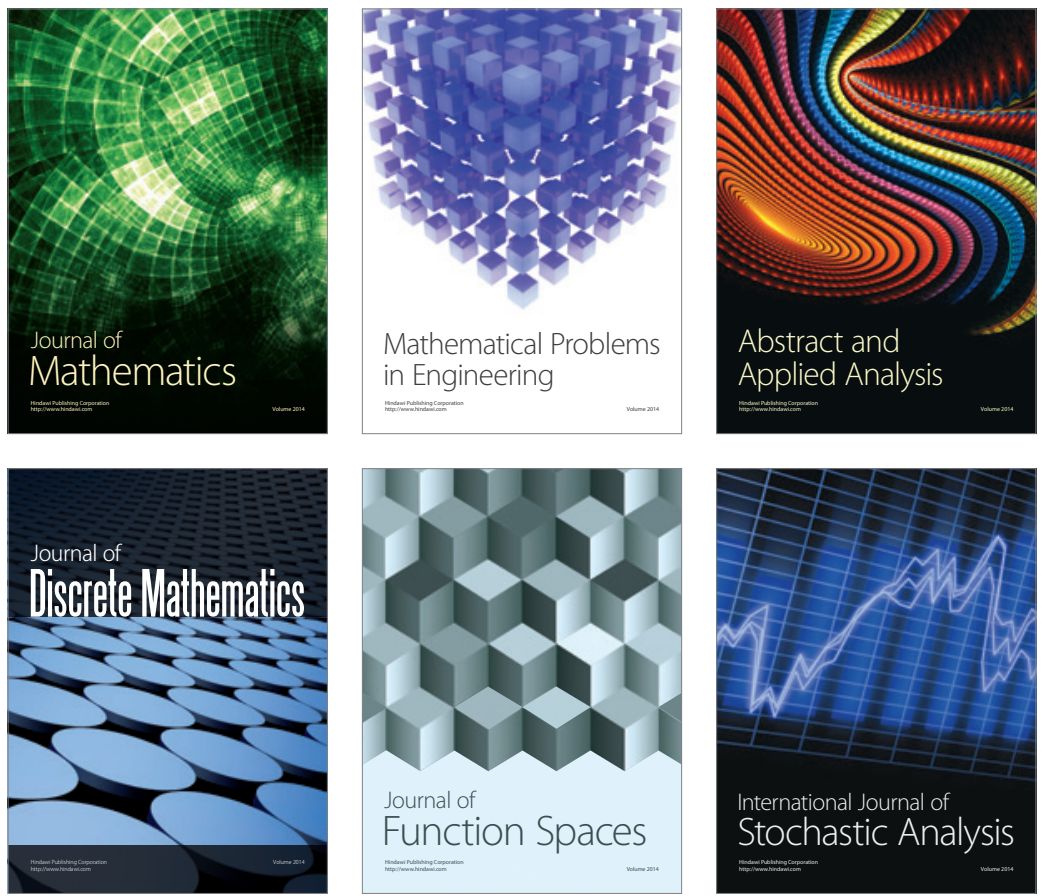

Journal of

Function Spaces

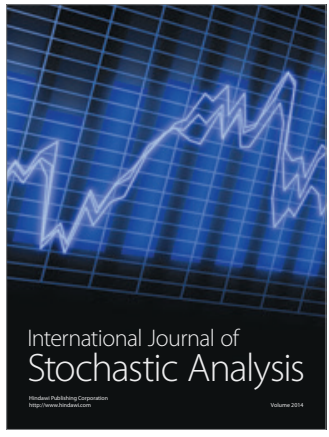

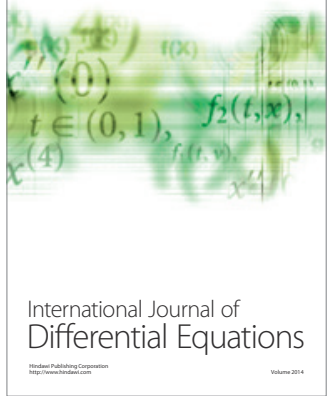
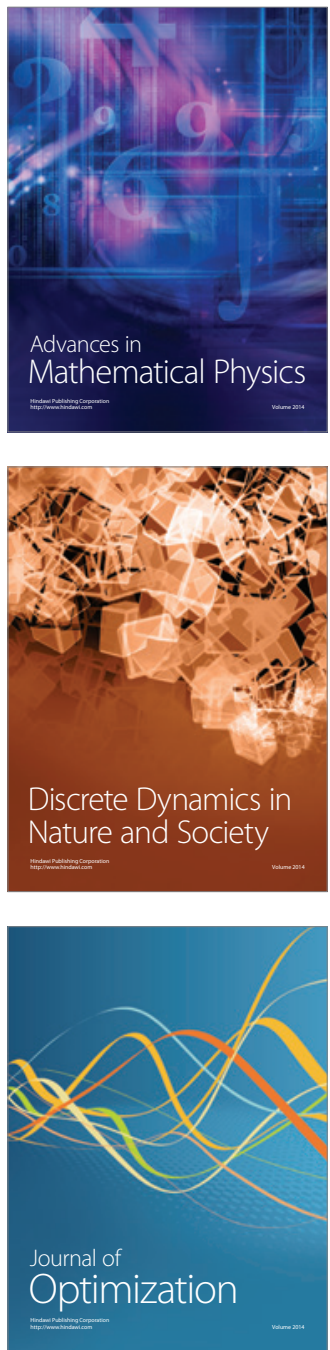